\title{
SIRT1 is a critical regulator of $K 562$ cell growth, survival, and differentiation
}

Mark T. Duncan ${ }^{1}$, Teresa A. DeLuca ${ }^{1}$, Hsin-Yu Kuo ${ }^{2,3,5}$, Minchang $\mathrm{Yi}^{4}$, Milan Mrksich ${ }^{2,3,5,6}$, and William M. Miller ${ }^{1,6}$

Departments of ${ }^{1}$ Chemical and Biological Engineering, ${ }^{2}$ Biomedical Engineering, and ${ }^{3}$ Chemistry, and ${ }^{4}$ Master of Biotechnology Program, Northwestern University, Evanston, Illinois 60208; ${ }^{5}$ Department of Cell and Molecular Biology and ${ }^{6}$ Robert H. Lurie Comprehensive Cancer Center, Northwestern University, Chicago, Illinois 60611

Correspondence: William M. Miller, Chemical and Biological Engineering Department, Northwestern University, 2145 Sheridan Rd., Tech E136, Evanston, IL, 60208, USA. E-mail: wmmiller@northwestern.edu

Key words: SIRT1, deacetylase, K562 cells

\section{Abstract}

Inhibition of histone deacetylases (HDACi) has emerged as a promising approach in the treatment of many types of cancer, including leukemias. Among the HDACs, Class III HDACs, also known as sirtuins (SIRTs), are unique in that their function is directly related to the cell's metabolic state through their dependency on the co-factor $\mathrm{NAD}^{+}$. In this study, we examined the relation between SIRTs and the growth, survival, and differentiation of K562 erythroleukemia cells. Using a mass spectrometry approach we previously developed, we show that SIRT expression and deacetylase activity in these cells changes greatly with differentiation state (undifferentiated vs. megakaryocytic differentiation vs. erythroid differentiation). Moreover, SIRT1 is crucially involved in regulating 
the differentiation state. Overexpression of wildtype (but not deacetylase mutant) SIRT1 resulted in upregulation of glycophorin A, $\sim 2$-fold increase in the mRNA levels of $\alpha, \gamma, \varepsilon$, and $\zeta$ - globins, and spontaneous hemoglobinization. Hemin-induced differentiation was also enhanced by (and depended on) higher SIRT1 levels. Since K562 cells are bipotent, we also investigated whether SIRT1 modulation affected their ability to undergo megakaryocytic (MK) differentiation. SIRT1 was required for commitment to the MK lineage and subsequent maturation, but was not directly involved in polyploidization of either K562 cells or an already-MK-committed cell line, CHRF288-11. The observed blockage in commitment to the MK lineage was associated with a dramatic decrease in the formation of autophagic vacuoles, which was previously shown to be required for K562 cell MK commitment. Autophagy-associated conversion of the protein LC3-I to LC3-II was greatly enhanced by overexpression of wildtype SIRT1, further suggesting a functional connection between SIRT1, autophagy, and MK differentiation. Based on its clear effects on autophagy, we also examined the effect of SIRT1 modulation on stress responses. Consistent with results of prior studies, we found that SIRT1 silencing modestly promoted drug-induced apoptosis, while overexpression was protective. Furthermore, pan-SIRT inhibition mediated by nicotinamide pretreatment substantially increased imatinib-induced apoptosis. Altogether, our results suggest a complex role for SIRT1 in regulating many aspects of K562 cell state and stress response. These observations warrant further investigation using normal and leukemic primary cell models. We further suggest that, ultimately, a well-defined mapping of HDACs to their substrates and corresponding signaling pathways will be important for optimally designing HDACi-based therapeutic approaches. 


\section{Introduction}

In recent years, lysine acetylation has come to be appreciated as a functionally important and pervasive regulator of protein activity. Acetylation can modify the activity of enzymes $[1,2]$, the localization and DNA-binding activity of transcription factors [3-6], availability of chromatin for transcription [7, 8], and protein stability [9]. In 2009, Choudary and colleagues utilized highresolution mass spectrometry to demonstrate over 3500 acetylation sites on 1750 proteins, affecting essentially every major cellular process [10]. Subsequent mass spectrometry-based studies have offered further evidence for the extensive role of acetylation in cell signaling [11-13]. Thus, it seems that many (if not most) proteins in the human cell are subject to acetylation and a host of other competing lysine modifications (e.g., ubiquination, sumoylation, etc.).

Within the cell, acetylation is mediated by histone acetyl transferases (HATs), and removal of the acetyl-group is catalyzed by histone deacetylases (HDACs). In human cells, there are 18 HDACs, which are grouped into 5 classes (I, IIa, IIb, III, IV), primarily based on their homology to yeast deacetylases. Class I, IIa, IIb, and IV HDACs are further known as the "classical" HDACs, as they retain significant commonalities with regards to structure and domain organization, exhibit $\mathrm{Zn}^{2+}$ dependence, and have similar inhibitor sensitivities [14]. In recent years, general inhibitors of the classical HDACs have received widespread attention for their ability to enhance chemotherapeutic drug efficacy in a wide variety of cell types in culture [15-17]. In addition, numerous classical HDAC inhibitors have undergone clinical trials for the treatment of leukemias and lymphomas, among many other cancer types [18]. Of particular note, vorinostat and romidepsin have already received FDA approval for treatment of cutaneous T-cell lymphomas. 
From a chemotherapeutic standpoint, less attention has been given to the Class III HDACs (also known as sirtuins (SIRTs)). SIRTs are unique in that their activity is directly linked to the real-time metabolic state of the cell through their dependence on $\mathrm{NAD}^{+}$as a co-factor. This is particularly interesting because of the long-speculated connection between cancer and metabolism dating back to Otto Warburg's observation in 1924 that cancer cells typically favor aerobic glycolysis over oxidative phosphorylation (recently reviewed by Vander Heiden et al. [19]). Because SIRTs appear to be designed for sensing the metabolic environment and responding in real-time, they could potentially serve as a transducer in implementing changes downstream of the altered metabolic state present in cancer cells. Interestingly, SIRT1 itself was recently shown to directly down-regulate glycolytic flux through deacetylation of the glycolytic enzyme phosphoglycerate mutase-1 [20]. Although this further complicates our understanding of the role of SIRTs, it demonstrates that there is substantial crosstalk between general cell metabolism and SIRT activities.

From a different standpoint, we and others have already provided evidence that SIRTs (especially SIRT1) can act as regulators of normal hematopoietic cell differentiation [21, 22]. Recent studies further suggest an important role for SIRT1 in hematopoietic stem cell (HSC) maintenance [23] and hematopoietic lineage specification [24]. Since leukemic cells are essentially undifferentiated progenitor cells with out-of-control proliferation (and a clearly failed terminal differentiation program), differentiation regulation could potentially represent another link between SIRTs and malignant phenotypes.

Based on the aforementioned points, we investigated whether SIRTs might be important for the phenotypic characteristics of chronic myeloid leukemia (CML) cells using the K562 cell line as a model system. We aimed to identify the relative contributions of SIRTs to the K562 cell's overall 
deacetylase activity, to investigate whether SIRT modulation might regulate the phenotype of K562 cells and, ultimately, have therapeutic implications for CML. Finally, we explored whether RNAi or inhibitor approaches could enhance apoptosis induced by imatinib, which is the current front-line treatment for bcr-abl+ CML.

\section{Materials and methods}

\section{Reagents}

Antibodies for flow cytometry (CD41, glycophorin A, and Annexin V) were from BD Biosciences. Chemical reagents were from Sigma Aldrich unless otherwise specified.

\section{Cell culture and differentiation}

K562 and CHRF-288-11 (CHRF) cells were maintained in Iscove's Modified Dulbecco's Media (IMDM) supplemented with 10\% fetal bovine serum (Hyclone). To induce megakaryocytic (MK) differentiation, cells were stimulated with $10 \mathrm{ng} / \mathrm{ml}$ phorbol 12-myristate 13-acetate (PMA). To induce erythroid (E) differentiation, K562 cells were treated with $30 \mu \mathrm{M}$ hemin.

\section{Preparation of lentiviral transfer vectors}

The original lentivectors used in the study (see Fig. 3A) were based on the pLVTHM vector [25] (Addgene \#12247) for gene silencing and the pWPI vector (Addgene \#12254) for overexpression. For creation of the shSIRT1 and shSIRT2 silencing vectors, oligonucleotides with overhangs corresponding to the MluI and ClaI restriction sites in PLVTHM were annealed and ligated into the digested pLVTHM vector. For creation of the SIRT1 overexpression vector, SIRT1 PCR product was cloned into pWPI at the PMEI site. A mutant SIRT1 (H363Y) based on the aforementioned construct was purchased from Genscript. 
For studies involving MK differentiation, we also made use of a purchased pGIPZ lentivector expressing a shRNAmir targeting SIRT1 (Open Biosystems, GE Healthcare) and, after validating the effectiveness of that shRNAmir, subsequently transferred it to the Tetracycline-inducible lentivector pTRIPZ (Open Biosystems) using standard cloning techniques.

\section{Virus production and transduction}

LentiX 293T cells (Clontech) were transfected with the packaging plasmids pMD2G, pSPAX2, and the relevant lentiviral transfer vector in a 3:8:10 mass ratio using Lipofectamine 2000 (Life Technologies). Lentivirus-containing supernatant was harvested $\sim 48 \mathrm{~h}$ after transfection and concentrated with PEG-it (Systems Biosciences) according to the manufacturer's recommendation. Briefly, supernatants were centrifuged at $500 \mathrm{x} \mathrm{g}, 4^{\circ} \mathrm{C}$, and clarified supernatant was then mixed with PEG-it and incubated overnight at $4^{\circ} \mathrm{C}$. The following day, precipitated lentivirus was collected by centrifugation at $1500 \mathrm{x}$ f for $30 \mathrm{~min}$, resuspended in phosphate buffered saline (PBS), aliquoted and stored at $-80^{\circ} \mathrm{C}$. Functional titers of virus were determined by transducing K562 cells with various dilutions of virus and subsequently analyzing GFP expression by flow cytometry.

For transduction, K562 or CHRF cells were spinoculated at a multiplicity of infection of $\sim 5$ for 30 min at $800 \mathrm{x} \mathrm{g}$ and $32^{\circ} \mathrm{C}$. In the case of pGIPZ-based lentiviruses, cells were then further selected with $1 \mu \mathrm{g} / \mathrm{ml}$ puromycin for at least 3 days to ensure a pure population. Although the pLVTHM and pWPI based lentiviruses do not contain an antibiotic selection marker, at the MOI used, transduction efficiencies were consistently $>90 \%$ (as determined by GFP expression). 


\section{Flow cytometry}

For analysis of surface antigen expression (CD41, GlyA), 25,000 - 50,000 cells were washed 2x with PBS supplemented with $0.5 \%$ bovine serum albumin (BSA) and $1 \mathrm{mM}$ EDTA (PEB). Then cells were incubated with antibody for 30 min at $4^{\circ} \mathrm{C}$, re-washed $2 x$ with PEB, and $10 \mu 1$ of DAPI $(70 \mu \mathrm{g} / \mathrm{ml})$ was added in order to identify non-viable cells. Alternatively, ploidy was analyzed by fixing PEB-washed cells with 0.5\% paraformaldehyde (PFA) for $15 \mathrm{~min}$ at room temperature (RT), followed by permeabilization with $70 \%$ methanol at $4^{\circ} \mathrm{C}$ for $1 \mathrm{~h}$. Cells were then washed $2 \mathrm{x}$ with $\mathrm{PEB}$, treated with RNAse for $30 \mathrm{~min}$ at $37^{\circ} \mathrm{C}$, and finally DNA was stained with propidium iodide $(50 \mu \mathrm{g} / \mathrm{ml})$. To determine ploidy in pTRIPZ-transduced cells, we performed Hoescht 33342 staining and analysis as previously described [26].

To evaluate apoptosis, cells were washed 2x with Annexin V Binding Buffer (BD Biosciences), stained with Annexin V and DAPI for $15 \mathrm{~min}$ at RT, and then analyzed. All samples were analyzed on an LSRII flow cytometer (BD Biosciences).

\section{cDNA synthesis and $q P C R$}

RNA was purified from cells using the RNAeasy kit (Qiagen) according to the manufacturer's instructions. RNA was then reverse transcribed into cDNA using Superscript III Reverse Transcriptase (Life Technologies). qPCR reactions were then prepared with Power Sybr Green Master Mix (BioRad), with 20 ng of cDNA as template per reaction. All reactions were performed in triplicate. qPCR primer sequences are provided in Supplemental Table 1. 


\section{Western Blotting}

K562 cells were collected before induction of differentiation, as well as three days after treatment with PMA or hemin, washed once with PBS, and frozen at $-80^{\circ} \mathrm{C}$. Cell lysates were prepared as previously described [12]. Lysates were diluted in $3 \mathrm{X}$ protein loading buffer (187.5 mM Tris (pH 6.8), $30 \%$ glycerol, $6 \%$ SDS, $0.005 \%$ pyronin $\mathrm{Y}, 5 \%$ beta-mercaptoethanol) and boiled for 5 minutes at $95^{\circ} \mathrm{C}$. Lysate proteins were separated by gel electrophoresis using Mini-PROTEAN TGX Precast Gels (BioRad), and transferred onto nitrocellulose membranes (BioRad). The membranes were blocked for $1 \mathrm{~h}$ at RT with $5 \%$ dried milk or 5\% BSA in TBST buffer (Trisbuffered saline $+0.1 \%$ Tween 20). Membranes were incubated with primary antibody in TBST + $5 \%$ milk or BSA with gentle shaking for $2 \mathrm{~h}$ at room temperature or overnight at $4{ }^{\circ} \mathrm{C}$. Membranes were washed and incubated with horseradish peroxidase (HRP)-conjugated anti-mouse- or antirabbit-IgG antibody (Cell Signaling). Protein was detected using Chemiluminescent HRP Substrate (Millipore) and film exposure. The SIRT1 (sc-74504), HSC70 (sc-7298), and SIRT7 (sc-135055) antibodies were obtained from Santa Cruz; SIRT2 (\#04-1124) and SIRT5 (\#ABE198) from Millipore; SIRT3 (\#5490) and SIRT6 (\#2590) from Cell Signaling.

\section{Analysis of HDAC activity by SAMDI}

The same lysate samples used for western blotting were used for analysis of HDAC activity. Lysates were diluted to a final protein concentration of $1 \mathrm{mg} / \mathrm{ml}$ in HDAC buffer $(25 \mathrm{mM}$ Tris $(\mathrm{pH}$ 8.0), $137 \mathrm{mM} \mathrm{NaCL}, 2.7 \mathrm{mM} \mathrm{KCl}, 1 \mathrm{mM} \mathrm{MgCl}_{2}$ ). For measuring sirtuin activity alone, trichostatin A (TSA) was added to the diluted lysates to a final concentration of $50 \mu \mathrm{M}$. For measuring class I/II/IV HDAC activity alone, nicotinamide (NIC) was added to the diluted lysates to a final concentration of $50 \mathrm{mM}$. Then, $5 \mu \mathrm{l}$ of the lysate was distributed into separate wells of a 384-well plate, and protease inhibitor cocktail (Roche) and $\mathrm{NAD}^{+}$were added to each well. Finally, the 
cysteine-terminated acetylated hexapeptide substrates were added at final concentration of $10 \mu \mathrm{M}$ to initiate the deacetylase reaction, and the plate was incubated at $37^{\circ} \mathrm{C}$ to allow the reaction to proceed. Eight different peptide substrates were used to profile activity, each with 3 or 4 replicate reactions per condition. After one hour, the reaction was quenched with deacetylase inhibitors, and $2 \mu \mathrm{l}$ of the reactions were transferred to an array plate. The array plate contained small, gold islands with a monolayer of alkanethiolates presenting maleimide groups to allow immobilization of the peptides in both the acetylated and deacetylated form. The array plate was then rinsed with deionized ultrafiltered water and ethanol, dried under nitrogen, and treated with matrix $(2,4,6-$ trihydroxyacetophenone, $20 \mathrm{mg} / \mathrm{mL}$ in acetone). The monolayers were analyzed by MALDI-TOF MS to obtain a mass spectrum for each spot. The peak areas for the acetylated and deacetylated forms of the peptide were integrated and used to calculate percent deacetylation (Deacetylase activity $=\boldsymbol{I}_{D} /\left(\boldsymbol{I}_{D}+\boldsymbol{I}_{A}\right)$ where subscript D refers to the deacetylated peptide product peak and A refers to the acetylated peptide substrate peak). Details of the mass spectrometry and peptide synthesis have been previously described [12].

\section{Analysis of PMA-induced autophagic vacuoles}

K562 cells stably transduced with a shRNAmir targeting SIRT1 or a non-silencing sequence were treated with $10 \mathrm{ng} / \mathrm{ml}$ PMA and cultured for the indicated times. Cells were collected, washed with PBS $+1 \%$ BSA, and cytospun onto glass slides. Cells were then stained with Wright-Giemsa (Camco) according to the manufacturer's instructions and imaged on a Leica wide-field microscope.

For quantification of vacuole formation, a researcher scored images after relabeling (in order to blind him with regards to the conditions). Cells were classified as having either (i) no vacuoles, (ii) small/punctate vacuoles, or (iii) large, non-punctate vacuoles that constituted a significant fraction of the cell body area. 


\section{Immunofluorescence}

Cells were fixed in situ with $4 \%$ PFA, washed with PBS, and permeabilized with $0.1 \%$ Triton X-

100. After blocking, cells were incubated with primary antibody against SIRT1 (Abcam \#32424) overnight at $4^{\circ} \mathrm{C}$. The following day, cells were washed, incubated with FITC-labeled secondary antibody for $2 \mathrm{~h}$, re-washed, and nuclei were labeled with DAPI. Samples were mounted and analyzed on a Leica SP5 confocal system.

\section{Results}

\section{Sirtuin expression and activity profile in $\mathrm{K562}$ cells depends on differentiation state}

In this study, we primarily focused on the CML line K562. K562 cells have some resemblance to the megakaryocytic-erythroid progenitors (MEPs) that have been hypothesized to give rise to both MK cells and red blood cells [27]. Treatment with hemin increases expression of the erythroidspecific antigen glycophorin A (GlyA) and stimulates hemglobinization of a large fraction of the cells. On the other hand, stimulation with PMA results in loss of GlyA expression (not shown), and acquisition of the MK antigen CD41, followed by polyploidization (Fig.1A).

Initially, we sought to characterize which SIRTs are expressed in these cells at each differentiation state. (Note that we neglected SIRT4 in some of the analyses, as it has been reported not to have any deacetylase activity [28].) SIRT1, 2, 3, 5, 6, and 7 were all readily detectable at the protein level in basal K562 cells (Fig. 1B). Several substantial changes in SIRT expression were observed after induction of differentiation with PMA or hemin. In the case of PMA, SIRT1/2/6/7 were strongly downregulated at the protein level, while SIRT3 was upregulated. In the case of hemin, the protein level of SIRT3 decreased dramatically, while the levels of SIRT1/6/7 decreased, but remained detectable. Because differences in antibody efficiency do not allow for a direct comparison 
between SIRT protein expression levels, we next sought to quantitate the mRNA expression of each SIRT. A copy number estimate for each SIRT was determined from a standard curve based on dilutions of linearized plasmid containing the corresponding cDNA (Fig. 1C). SIRT1 and SIRT2 mRNAs were expressed at significantly higher levels compared to SIRT3-7. PMA decreased SIRT1/2 50\%. However, besides this, relatively small changes in mRNA levels were observed in response to either hemin or PMA treatment.

Next, we examined the relative importance of SIRT deacetylase activity in comparison to class I/II/IV HDACs. To do this, we utilized Self-Assembled Monolayers and matrix-assisted laser Desorption Ionization time-of-flight mass spectrometry (SAMDI), as we have previously described. This assay consists of incubating a panel of short, acetylated peptides with cell lysate, followed by covalent attachment to a self-assembled monolayer of alkane-thiolates on a gold surface, and then the proportion of peptide that has been deacetylated is quantified by mass spectrometry. The 8 peptide substrates chosen were shown to be substrates for human deacetylases in earlier work [12, 29, 30]. The total cell lysate reveals deacetylase activity contributed from both SIRTs and the class I/II/IV HDACs. To selectively gauge the level of SIRT deacetylase activity alone, the peptidelysate incubation is performed in the presence of TSA, a well-known generic class I/II/IV HDAC inhibitor. Alternatively, to determine class I/II/IV HDAC activity, peptide-lysate incubations were performed in the presence of the pan-SIRT inhibitor NIC.

Interestingly, we found that SIRTs provided the dominant deacetylase activity in undifferentiated and hemin-differentiated K562 cells (Fig. 2). The class I/II/IV HDACs (+NIC condition) showed little change in activity with either PMA- or hemin-mediated differentiation. Notably, the SIRT activity (+TSA condition) was dramatically decreased in PMA-induced MK differentiation, but 
SIRT activity remained comparatively unchanged with hemin-induced differentiation. These activity trends are consistent with protein expression patterns of many of the SIRT deacetylases

(Fig. 1B). The decrease in SIRT activity with PMA differentiation is consistent with our earlier report examining a different leukemic cell line, $\mathrm{CHRF}$, that is already committed to the MK lineage [12]. This suggests that there may be a significant relationship between E/MK specification and SIRT activity, and especially SIRT1/2, as these were the most highly expressed isoforms at the time of inducing differentiation. The rise in SIRT3 protein levels observed for PMA-induced MK differentiation (Fig. 1B) was also seen with CHRF cells and suggests a potentially lineage-specific role. Regarding classical HDAC activity, there seemed to be a modest increase in deacetylation for most peptides following treatment with PMA.

\section{SIRT1 is a key regulator of K562 cell growth and erythroid differentiation}

Multiple previous reports have shown that SIRT1 is involved in hematopoietic stem cell maintenance [23], as well as cell differentiation in multiple tissue types [31, 32]. Based on this and the aforementioned results indicating high levels of SIRT1/2 activity, we decided to examine potential roles for SIRT1 in regulating the phenotype of K562 cells. We constructed and introduced lentivectors for either silencing SIRT1 or overexpression of wildtype or deacetylase mutant SIRT1 (Fig. 3A). Silencing was $\sim 85 \%$ efficient and the overexpression level was $\sim 200$-fold that of the control by qPCR (Fig. 3B). Interestingly, we found that the growth rate of undifferentiated K562 cells was dramatically reduced with the introduction of wildtype SIRT1 and modestly reduced by mutant SIRT1 (Fig. 3C).

Because of this, we further investigated whether the erythroid-like phenotype of the cells had been altered, as assessed by expression of GlyA and CD71. Although CD71 expression was relatively 
unchanged (not shown), GlyA expression was dramatically increased in cells overexpressing wildtype (but not mutant) SIRT1 (Fig. 4A). We thus hypothesized that high SIRT1 levels may be driving terminal erythroid differentiation. Consistent with this, we found that $\sim 15 \%$ of overexpressing cells were already hemoglobin positive without any differentiation stimulus. Under hemin stimulation, these cells also achieved a much greater extent of hemoglobinization compared to the empty vector control (62\% vs $26 \%$ at Day 3) (Fig. 4B). This effect was again dependent on SIRT1's deacetylase activity. Consistently, we observed a substantial reduction in hemoglobinization with SIRT1 silencing. Although SIRT2 has substantial substrate overlap with SIRT1 [33] and there is evidence for situational functional redundancy [34], silencing SIRT2 did not affect erythroid differentiation of K562 cells (Fig. S1A,B).

Next, we examined the effect of SIRT1 modulation on globin mRNA. Xue et al. previously reported that SIRT1 positively regulates $\varepsilon$-globin expression through SATB1 during erythroid differentiation [22]. We also observed this effect, and further observed similar effects for $\alpha-, \zeta-$, and $\gamma$-globins (Fig. 4C). Overexpression of wildtype SIRT1 resulted in $\sim 2$-fold upregulation of each globin mRNA. Consistently, silencing resulted in a 30-40\% reduction in globin levels compared to the non-silencing control, and overexpression of mutant SIRT1 reduced $\varepsilon^{-}, \gamma_{-}$, and $\zeta$-globins by 30$50 \%$ compared to the empty vector control. Thus, globin expression clearly depends on the level of SIRT1 deacetylase activity. Taken together, our results demonstrate widespread effects of SIRT1 driving cells towards a more mature erythroid differentiation state.

\section{SIRT1 also regulates PMA-induced MK commitment, but not PMA-induced polyploidization}

We next examined whether SIRT1 was also required for PMA-induced MK differentiation of K562 cells. To avoid the changes in phenotype induced by changing SIRT1 level prior to the initiation of 
differentiation, we utilized a quasi-inducible lentivector wherein a shRNAmir targeting SIRT1 is driven by the CMV promoter. The CMV promoter has only weak activity in K562 cells until it is activated by treatment with PMA. Thus, this vector caused only weak silencing until after induction with PMA (Fig. S2A).

As in erythroid differentiation, we found that K562 cells lacking SIRT1 could not efficiently commit to the megakaryocytic lineage as assessed by CD41 expression (Fig. 5A), but SIRT2 silencing had no effect (Fig. S1C). Expectedly, the fraction of cells that subsequently became polyploid was also greatly reduced by shSIRT1 (Fig. 5B). Overexpression of the deacetylase mutant SIRT1 resulted in similar defects in differentiation as SIRT1 silencing (data not shown Fig. S3). The reduction in polyploidization mediated by silencing could not be attributed to differences in cell proliferation, as both control and shSIRT1-expressing cells continued to divide after PMA treatment, but by Day 3 expansion had stopped and cell number began to decline (Fig. S2B). We analyzed DNA synthesis by BrdU incorporation after 1 and 3 days of PMA treatment (Fig. S2C), and found that shSIRT1 did cause a clear reduction in the number of endomitotic cells (BrdU+ and $\geq 4 \mathrm{~N}$ DNA content) at Day 3, when polyploidization is first becoming apparent (see Fig. 5B). We also investigated changes in the expression of genes related to MK maturation/polyploidization: cyclin D1, cyclin D3, and p21. In primary megakaryocytes cyclin D3 is known to be a key driver of endomitotic cell cycling [35], while PMA-induced MK differentiation in K562 cells corresponds to dramatic increases in cyclin D1 and p21 [36]. Consistent with a reduction in cell cycling, we found that SIRT1 silencing resulted in a $130 \%$ increase in p21 levels (relative to the non-silencing control) after 3 days of PMA treatment (Fig. S2D). We note that the effects on p21 levels were not mediated through p53 (a well-known SIRT1 substrate), since K562 cells are p53 null. 
We and others have previously provided evidence that SIRT1 opposes polyploidization in primary MK cells and endothelial cells $[21,37,38]$. Thus, we investigated the effects of SIRT1 on PMAinduced polyploidization in greater detail. First, we examined the committed MK cell line CHRF, which expresses the immature MK marker CD41, but not the more mature marker CD42 (Fig. 6A). We found that silencing SIRT1 did not affect the polyploidization of these cells (Fig. 6B). Next, we used a doxycycline-inducible lentivector to reexamine the effects of SIRT1 silencing in K562 cells. In this experiment, we induced silencing by doxycycline (dox) addition at various times relative to the induction of MK differentiation by PMA addition (Fig. 6C). Adding dox $<2$ days after PMA treatment notably reduced the ploidy, but adding it at later times (e.g., day 4 or 5 of PMA treatment), when cells have largely already committed to the MK lineage, did not substantially alter the polyploidization versus the - dox control (Fig. 6D). Taken together, these results show SIRT1 is not substantially involved in PMA-induced polyploidization.

\section{SIRT1 may regulate MK differentiation of K562 cells through enhancing PMA-induced autophagy}

Concomitant with reduced MK commitment, we observed a dramatic reduction in PMA-induced autophagic vacuole formation in K562 cells with SIRT1 silencing, and an increase in vacuole formation with overexpression (Fig. 7A,B). Moreover, despite its often cited nuclear localization, a substantial fraction of SIRT1 was found in the cytosol in K562 cells (Fig. 8A). This indicates a possible mechanism by which SIRT1 depletion blocks K562 cell commitment to the MK lineage, as autophagy has been shown to be essential for PMA-induced MK commitment for these cells [39]. SIRT1 is known to affect autophagy in other cell types, and one of the mechanisms appears to be deacetylation of ATG family proteins [40]. To confirm that SIRT1 was affecting PMA-induced autophagy in K562 cells, we checked conversion of LC3-I to LC3-II (a commonly used marker of 
autophagy induction [41]) after $6 \mathrm{~h}$ of stimulation with PMA. We also co-treated cells with chloroquine (CQ) to block degradation of autophagasomes in order to better observe changes in the autophagic flux over the treatment period. We found that cells overexpressing wildtype SIRT1 showed a dramatic increase in LC3-II compared to the control (Fig. 8B).

\section{Nicotinamide pre-treatment enhances imatinib-induced apoptosis}

Based on the fact that SIRT1 can promote autophagy in K562 cells, we also considered whether it might affect apoptosis. The relationship between autophagy and apoptosis is complex, and has often generated conflicting reports [42-46]. However, most literature examining CML cells or leukemic cell lines, such as K562, indicate that inhibition of autophagy tends to promote apoptosis [47]. In fact, the autophagy inhibitor hydroxychloroquine (HCQ) is currently in clinical trials to examine whether co-treatment with HCQ and imatinib offers a therapeutic benefit over imatinib alone [48].

While this work was in progress, it was reported that SIRT1 silencing does indeed enhance apoptosis induced by imatinib in primary CML [49] and K562 cells [50]. We observed the same tendency, although with a more modest enhancement with shSIRT1 (Fig. 9A). This could be due to differences in silencing efficiency, the RNAi approach used, or other experimental variables. Because there may be some functional redundancy between SIRTs, we also examined the effects of NIC with and without imatinib. NIC alone reduced cell growth at doses greater than $12.5 \mathrm{mM}$, but toxicity as determined by AnnexinV/DAPI staining was only apparent at $50 \mathrm{mM}$ (Fig. 9B,C). Because of that, we examined NIC concentrations ranging from $1 \mathrm{mM}$ to $25 \mathrm{mM}$ in combination with $1 \mu \mathrm{M}$ imatinib. Although simultaneous treatment with NIC and imatinib did not enhance apoptosis, performing a $24 \mathrm{~h}$ pretreatment with $25 \mathrm{mM}$ NIC did enhance imatinib-induced apoptosis 
(Fig. 9D). These results suggest that, although NIC generally restrains K562 cell growth, changes in the cell state (epigenetic? metabolic?) are required for an enhanced apoptotic response to imatinib.

We also tested whether SIRT1 would affect apoptosis induced by a drug with a completely different mechanism of action. Actinomycin D (ActD) blocks both RNA PolI- and PolII-mediated transcription at high doses $(\sim \mu \mathrm{g} / \mathrm{ml}$ range $)$, is selective for Poll at lower doses $(\sim \mathrm{ng} / \mathrm{ml}$ range $)$ [51] and rapidly induces apoptosis in K562 cells [52]. We found that SIRT1 was strongly protective against ActD-induced apoptosis (Fig. S4), while silencing modestly enhanced apoptosis. Since ActD ultimately acts to reduce de novo protein synthesis (especially through inhibition of PolImediated rRNA transcription), we speculate that SIRT1's well-known activation of the heat shock pathway may be responsible for sustaining viability in cells with reduced capability for protein synthesis [53].

\section{Discussion}

In this study, we have demonstrated the importance of SIRT1 activity for the maintenance of the normal K562 cell phenotype and differentiation potential. This is consistent with the recent literature indicating an important role for SIRT1 in HSC and embryonic stem cell (ESC) maintenance $[23,54,55]$. Additionally, although there are obvious differences in the E/MK differentiation programs of normal HSCs and K562 cells, there are also substantial overlaps [5660], suggesting SIRT1 may have important involvement in normal E/MK differentiation. This deserves further investigation. 
In any case, it is clear that SIRT1 is a crucial player in the regulation of autophagy and the cell's stress response. In light of this, it would seem that SIRT1 (and perhaps other SIRTs) are natural targets for therapeutic intervention. Although preliminary, our results with NIC/imatinib cotreatment are particularly interesting because (i) NIC is already used for various skin and anxiety disorders [61, 62] and (ii) NIC is well-tolerated at high intravenous doses [63].

From a broad perspective, it should be said that, despite intensive study in recent years, we still lack a precise understanding of the totality of SIRTs' functions and this clearly impedes its therapeutic exploitation. This in large part stems from the vast number of proteins and signaling pathways regulated by SIRTs, making their predominant effects highly dependent on the cellular context. For instance, although SIRT1 is normally thought of as anti-apoptotic, cytoplasmic SIRT1 actually promotes apoptosis in HeLa cells [64] and SIRT1 unexpectedly promotes mouse ESC apoptosis mediated by endogenous reactive oxygen species [65]. Seeming contradictions are also reported with regards to hematopoiesis. For instance, SIRT1 was shown to be important for HSC maintenance using a mouse knockout model [23], but inhibiting SIRTs by treating CD34+ cells from umbilical cord blood with NIC promoted HSC self-renewal while opposing differentiation [66]. Discrepancies like this could occur for many reasons: the physiological difference between SIRT1 absence and inhibition, potential functional redundancy between SIRTs, additional effects of NIC (e.g., increasing [NAD $\left.{ }^{+}\right][21]$, inhibiting other NAD ${ }^{+}$-dependent enzymes such as PARP [67]), etc. Also, although a diverse set of SIRT inhibitors has been described, the lack of inhibitors with high specificity and effectiveness remains a critical issue [68].

Our ability to understand and predict the effects of both SIRT and classical HDAC inhibitors would certainly improve if we possessed a detailed understanding of each HDAC's binding partners, 
substrates, and corresponding signaling pathways. Given the thousands of proteins in a cell, this is impossible to achieve with any kind of expediency using traditional assays. Thus, we believe that development and application of new technologies (especially mass-spectrometry-based methods, such as SAMDI) and computational approaches will be especially useful in mapping out the overlapping and opposing effects of the 18 human HDACs. We anticipate that this "map", in combination with the development of more specific HDAC inhibitors, will allow for tailoring combinatorial HDACi treatments specific to a particular malignancy.

\section{Acknowledgments}

This work was supported by grants from the National Institutes of Health (R01HL093083, WM and R21DE024388, MM) and the National Science Foundation (CBET 0853603, WM). MTD was supported in part by NIH predoctoral Biotechnology Training Grant T32GM008449 and TAD was supported in part by NIH/NCI training grant T32CA09560. This work was supported by the Northwestern University Flow Cytometry Facility and a Cancer Center Support Grant (NCI CA060553), and also the Northwestern University Biological Imaging Facility. We thank Lily Helfrich and Ahmad Malik for their assistance with a few experiments.

\section{References}

[1] Q. Wang, Y. Zhang, C. Yang, H. Xiong, Y. Lin, J. Yao, H. Li, L. Xie, W. Zhao, Y. Yao, Z.B. Ning, R. Zeng, Y. Xiong, K.-L. Guan, S. Zhao, G.-P. Zhao, Acetylation of Metabolic Enzymes Coordinates Carbon Source Utilization and Metabolic Flux, Science 327 (2010) 1004-1007. 
[2] V.J. Starai, I. Celic, R.N. Cole, J.D. Boeke, J.C. Escalante-Semerena, Sir2-Dependent Activation of Acetyl-CoA Synthetase by Deacetylation of Active Lysine, Science 298 (2002) 2390-2392.

[3] J. Luo, A.Y. Nikolaev, S.-i. Imai, D. Chen, F. Su, A. Shiloh, L. Guarente, W. Gu, Negative Control of p53 by Sir2 $\alpha$ Promotes Cell Survival under Stress, Cell 107 (2001) 137-148.

[4] A. Brunet, L.B. Sweeney, J.F. Sturgill, K.F. Chua, P.L. Greer, Y. Lin, H. Tran, S.E. Ross, R. Mostoslavsky, H.Y. Cohen, L.S. Hu, H.-L. Cheng, M.P. Jedrychowski, S.P. Gygi, D.A. Sinclair, F.W. Alt, M.E. Greenberg, Stress-Dependent Regulation of FOXO Transcription Factors by the SIRT1 Deacetylase, Science 303 (2004) 2011-2015.

[5] Y. Yang, H. Hou, E.M. Haller, S.V. Nicosia, W. Bai, Suppression of FOXO1 activity by FHL2 through SIRT1-mediated deacetylation, EMBO J 24 (2005) 1021-1032.

[6] J. Yuan, K. Minter-Dykhouse, Z. Lou, A c-Myc-SIRT1 feedback loop regulates cell growth and transformation, The Journal of Cell Biology 185 (2009) 203-211.

[7] D.Y. Lee, J.J. Hayes, D. Pruss, A.P. Wolffe, A positive role for histone acetylation in transcription factor access to nucleosomal DNA, Cell 72 (1993) 73-84.

[8] J.D. Anderson, P.T. Lowary, J. Widom, Effects of histone acetylation on the equilibrium accessibility of nucleosomal DNA target sites, Journal of Molecular Biology 307 (2001) 977-985.

[9] C. Caron, C. Boyault, S. Khochbin, Regulatory cross-talk between lysine acetylation and ubiquitination: role in the control of protein stability, BioEssays 27 (2005) 408-415.

[10] C. Choudhary, C. Kumar, F. Gnad, M.L. Nielsen, M. Rehman, T.C. Walther, J.V. Olsen, M. Mann, Lysine Acetylation Targets Protein Complexes and Co-Regulates Major Cellular Functions, Science 325 (2009) 834-840.

[11] C. Yi, M. Ma, L. Ran, J. Zheng, J. Tong, J. Zhu, C. Ma, Y. Sun, S. Zhang, W. Feng, L. Zhu, Y. Le, X. Gong, X. Yan, B. Hong, F.-J. Jiang, Z. Xie, D. Miao, H. Deng, L. Yu, Function and Molecular Mechanism of Acetylation in Autophagy Regulation, Science 336 (2012) 474-477.

[12] H.-Y. Kuo, T.A. DeLuca, W.M. Miller, M. Mrksich, Profiling Deacetylase Activities in Cell Lysates with Peptide Arrays and SAMDI Mass Spectrometry, Analytical Chemistry 85 (2013) 10635-10642.

[13] M. Bennetzen, D. Larsen, C. Dinant, S. Watanabe, J. Bartek, J. Lukas, J.S. Andersen, Acetylation dynamics of human nuclear proteins during the ionizing radiation-induced DNA damage response, Cell Cycle 12 (2013) 1688-1695.

[14] X.J. Yang, E. Seto, HATs and HDACs: from structure, function and regulation to novel strategies for therapy and prevention, Oncogene 26 (2007) 5310-5318.

[15] M.S. Kim, M. Blake, J.H. Baek, G. Kohlhagen, Y. Pommier, F. Carrier, Inhibition of Histone Deacetylase Increases Cytotoxicity to Anticancer Drugs Targeting DNA, Cancer Research 63 (2003) 7291-7300.

[16] T.K. Nguyen, E. Attkisson, Y. Dai, L. Kramer, M. Muschen, S. Grant, Abstract 2572: The histone deacetylase inhibitors (HDACIs) vorinostat and entinostat interact synergistically with the Bcr/Abl, FLT3, and aurora kinase inhibitor KW-2449 to induce apoptosis in imatininb mesylate (IM)-sensitive and -resistant CML and ALL cells in vitro and in vivo, Cancer Research 71 (2011) 2572.

[17] C. Yang, E. Choy, F.J. Hornicek, K.B. Wood, J.H. Schwab, X. Liu, H. Mankin, Z. Duan, Histone deacetylase inhibitor (HDACI) PCI-24781 enhances chemotherapy induced apoptosis in multidrug resistant sarcoma cell lines, Anticancer research 31 (2011) 11151123. 
[18] K. Keller, M. Jung, Histone Deacetylase (HDAC) Inhibitors in Recent Clinical Trials for Cancer Therapy, in: M. Lübbert, P.A. Jones (Eds.), Epigenetic Therapy of Cancer, Springer Berlin Heidelberg, 2014, pp. 227-255.

[19] M.G. Vander Heiden, L.C. Cantley, C.B. Thompson, Understanding the Warburg Effect: The Metabolic Requirements of Cell Proliferation, Science 324 (2009) 1029-1033.

[20] W.C. Hallows, W. Yu, J.M. Denu, Regulation of Glycolytic Enzyme Phosphoglycerate Mutase-1 by Sirt1 Protein-mediated Deacetylation, Journal of Biological Chemistry 287 (2012) 3850-3858.

[21] L.M. Giammona, S. Panuganti, J.M. Kemper, P.A. Apostolidis, S. Lindsey, E.T. Papoutsakis, W.M. Miller, Mechanistic studies on the effects of nicotinamide on megakaryocytic polyploidization and the roles of NAD+ levels and SIRT inhibition, Experimental Hematology 37 (2009) 1340-1352.e1343.

[22] Z. Xue, X. Lv, W. Song, X. Wang, G.-N. Zhao, W.-T. Wang, J. Xiong, B.-B. Mao, W. Yu, B. Yang, J. Wu, L.-Q. Zhou, D.-L. Hao, W.-J. Dong, D.-P. Liu, C.-C. Liang, SIRT1 deacetylates SATB1 to facilitate MARHS2-MAR $\varepsilon$ interaction and promote $\varepsilon$-globin expression, Nucleic Acids Research 40 (2012) 4804-4815.

[23] K. Matsui, S. Ezoe, K. Oritani, M. Shibata, M. Tokunaga, N. Fujita, A. Tanimura, T. Sudo, H. Tanaka, M.W. McBurney, I. Matsumura, Y. Kanakura, NAD-dependent histone deacetylase, SIRT1, plays essential roles in the maintenance of hematopoietic stem cells, Biochemical and Biophysical Research Communications 418 (2012) 811-817.

[24] P. Rimmelé, Carolina L. Bigarella, R. Liang, B. Izac, R. Dieguez-Gonzalez, G. Barbet, M. Donovan, C. Brugnara, Julie M. Blander, David A. Sinclair, S. Ghaffari, Aging-like Phenotype and Defective Lineage Specification in SIRT1-Deleted Hematopoietic Stem and Progenitor Cells, Stem Cell Reports 3 (2014) 44-59.

[25] M. Wiznerowicz, D. Trono, Conditional Suppression of Cellular Genes: Lentivirus VectorMediated Drug-Inducible RNA Interference, Journal of Virology 77 (2003) 8957-8951.

[26] Y. Ojima, M.T. Duncan, R.W. Nurhayati, M. Taya, W.M. Miller, Synergistic effect of hydrogen peroxide on polyploidization during the megakaryocytic differentiation of K562 leukemia cells by PMA, Experimental Cell Research 319 (2013) 2205-2215.

[27] N. Debili, L. Coulombel, L. Croisille, A. Katz, J. Guichard, J. Breton-Gorius, W. Vainchenker, Characterization of a bipotent erythro-megakaryocytic progenitor in human bone marrow, Blood 88 (1996) 1284-1296.

[28] N. Ahuja, B. Schwer, S. Carobbio, D. Waltregny, B.J. North, V. Castronovo, P. Maechler, E. Verdin, Regulation of Insulin Secretion by SIRT4, a Mitochondrial ADPribosyltransferase, Journal of Biological Chemistry 282 (2007) 33583-33592.

[29] Z.A. Gurard-Levin, J. Kim, M. Mrksich, Combining Mass Spectrometry and Peptide Arrays to Profile the Specificities of Histone Deacetylases, ChemBioChem 10 (2009) 2159-2161.

[30] Z.A. Gurard-Levin, K.A. Kilian, J. Kim, K. Bähr, M. Mrksich, Peptide Arrays Identify Isoform-Selective Substrates for Profiling Endogenous Lysine Deacetylase Activity, ACS Chemical Biology 5 (2010) 863-873.

[31] C.-M. Bäckesjö, Y. Li, U. Lindgren, L.-A. Haldosén, Activation of Sirt1 Decreases Adipocyte Formation During Osteoblast Differentiation of Mesenchymal Stem Cells, Journal of Bone and Mineral Research 21 (2006) 993-1002.

[32] S. Hisahara, S. Chiba, H. Matsumoto, M. Tanno, H. Yagi, S. Shimohama, M. Sato, Y. Horio, Histone deacetylase SIRT1 modulates neuronal differentiation by its nuclear translocation, Proceedings of the National Academy of Sciences 105 (2008) 15599-15604.

[33] P. Martinez-Redondo, A. Vaquero, The diversity of histone versus nonhistone sirtuin substrates, Genes Cancer 4 (2013) 148-163. 
[34] B. Peck, C.-Y. Chen, K.-K. Ho, P. Di Fruscia, S.S. Myatt, R.C. Coombes, M.J. Fuchter, C.D. Hsiao, E.W.-F. Lam, SIRT Inhibitors Induce Cell Death and p53 Acetylation through Targeting Both SIRT1 and SIRT2, Molecular Cancer Therapeutics 9 (2010) 844-855.

[35] J.M. Zimmet, D. Ladd, C.W. Jackson, P.E. Stenberg, K. Ravid, A role for cyclin D3 in the endomitotic cell cycle, Molecular and Cellular Biology 17 (1997) 7248-7259.

[36] C. Tringali, B. Lupo, F. Cirillo, N. Papini, L. Anastasia, G. Lamorte, P. Colombi, R. Bresciani, E. Monti, G. Tettamanti, B. Venerando, Silencing of membrane-associated sialidase Neu3 diminishes apoptosis resistance and triggers megakaryocytic differentiation of chronic myeloid leukemic cells K562 through the increase of ganglioside GM3, Cell Death and Differentiation 16 (2008) 164-174.

[37] L.M. Giammona, P.G. Fuhrken, E.T. Papoutsakis, W.M. Miller, Nicotinamide (vitamin B3) increases the polyploidisation and proplatelet formation of cultured primary human megakaryocytes, British Journal of Haematology 135 (2006) 554-566.

[38] N.M. Borradaile, J.G. Pickering, Polyploidy impairs human aortic endothelial cell function and is prevented by nicotinamide phosphoribosyltransferase, Am J Physiol Cell Physiol 298 (2010) C66-74.

[39] P. Colosetti, A. Puissant, G. Robert, F. Luciano, A. Jacquel, P. Gounon, J.-P. Cassuto, P. Auberger, Autophagy is an important event for megakaryocytic differentiation of the chronic myelogenous leukemia K562 cell line, Autophagy 5 (2009) 1092-1098.

[40] I.H. Lee, L. Cao, R. Mostoslavsky, D.B. Lombard, J. Liu, N.E. Bruns, M. Tsokos, F.W. Alt, T. Finkel, A role for the NAD-dependent deacetylase Sirt1 in the regulation of autophagy, Proceedings of the National Academy of Sciences 105 (2008) 3374-3379.

[41] N. Mizushima, T. Yoshimorim, B. Levine, Methods in Mammalian Autophagy Research, Cell 140 (2010) 313-326.

[42] C. Bellodi, M.R. Lidonnici, A. Hamilton, G.V. Helgason, A.R. Soliera, M. Ronchetti, S. Galavotti, K.W. Young, T. Selmi, R. Yacobi, R.A. Van Etten, N. Donato, A. Hunter, D. Dinsdale, Tirr, xF, Elena, P. Vigneri, P. Nicotera, M.J. Dyer, T. Holyoake, P. Salomoni, B. Calabretta, Targeting autophagy potentiates tyrosine kinase inhibitor-induced cell death in Philadelphia chromosome-positive cells, including primary CML stem cells, The Journal of Clinical Investigation 119 (2009) 1109-1123.

[43] Y. Kamitsuji, J. Kuroda, S. Kimura, S. Toyokuni, K. Watanabe, E. Ashihara, H. Tanaka, Y. Yui, M. Watanabe, H. Matsubara, Y. Mizushima, Y. Hiraumi, E. Kawata, T. Yoshikawa, T. Maekawa, T. Nakahata, S. Adachi, The Bcr-Abl kinase inhibitor INNO-406 induces autophagy and different modes of cell death execution in Bcr-Abl-positive leukemias, Cell Death Differ 15 (2008) 1712-1722.

[44] Y. Yu, L. Yang, M. Zhao, S. Zhu, R. Kang, P. Vernon, D. Tang, L. Cao, Targeting microRNA-30a-mediated autophagy enhances imatinib activity against human chronic myeloid leukemia cells, Leukemia 26 (2012) 1752-1760.

[45] B.M. Elzinga, M.J. Nyhan, L.C. Crowley, T.R. O'Donovan, M.R. Cahill, S.L. McKenna, Induction of autophagy by Imatinib sequesters Bcr-Abl in autophagosomes and downregulates Bcr-Abl protein, American Journal of Hematology 88 (2013) 455-462.

[46] Y. Mishima, Y. Terui, Y. Mishima, A. Taniyama, R. Kuniyoshi, T. Takizawa, S. Kimura, K. Ozawa, K. Hatake, Autophagy and autophagic cell death are next targets for elimination of the resistance to tyrosine kinase inhibitors, Cancer Science 99 (2008) 2200-2208.

[47] G.V. Helgason, M. Karvela, T.L. Holyoake, Kill one bird with two stones: potential efficacy of BCR-ABL and autophagy inhibition in CML, Blood 118 (2011) 2035-2043. 
[48] A. Mukhopadhyay, G.V. Helgason, M. Karvela, T.L. Holyoake, Hydroxychloroquine for chronic myeloid leukemia: complete cure on the horizon?, Expert Rev Hematol. 4 (2011) 369-371.

[49] L. Li, L. Wang, L. Li, Z. Wang, Y. Ho, T. McDonald, Tessa L. Holyoake, W. Chen, R. Bhatia, Activation of p53 by SIRT1 Inhibition Enhances Elimination of CML Leukemia Stem Cells in Combination with Imatinib, Cancer cell 21 (2012) 266-281.

[50] H. Yuan, Z. Wang, L. Li, H. Zhang, H. Modi, D. Horne, R. Bhatia, W. Chen, Activation of stress response gene SIRT1 by BCR-ABL promotes leukemogenesis, Blood 119 (2012) 1904-1914.

[51] O. Bensaude, Inhibiting eukaryotic transcription: Which compound to choose? How to evaluate its activity?, Transcription 2 (2011) 103-108.

[52] U. Ziegler, P. Groscurth, Morphological features of cell death, News Physiol Sci 19 (2004) 124-128.

[53] S.D. Westerheide, J. Anckar, S.M. Stevens, L. Sistonen, R.I. Morimoto, Stress-Inducible Regulation of Heat Shock Factor 1 by the Deacetylase SIRT1, Science 323 (2009) 10631066.

[54] S.K. Singh, C.A. Williams, K. Klarmann, S.S. Burkett, J.R. Keller, P. Oberdoerffer, Sirt1 ablation promotes stress-induced loss of epigenetic and genomic hematopoietic stem and progenitor cell maintenance, The Journal of Experimental Medicine 210 (2013) 987-1001.

[55] X. Ou, H.D. Chae, R.H. Wang, W.C. Shelley, S. Cooper, T. Taylor, Y.J. Kim, C.X. Deng, M.C. Yoder, H.E. Broxmeyer, SIRT1 deficiency compromises mouse embryonic stem cell hematopoietic differentiation, and embryonic and adult hematopoiesis in the mouse, Blood 117 (2011) 440-450.

[56] K.E. Elagib, F.K. Racke, M. Mogass, R. Khetawat, L.L. Delehanty, A.N. Goldfarb, RUNX1 and GATA-1 coexpression and cooperation in megakaryocytic differentiation, Blood 101 (2003) 4333-4341.

[57] M. Eisbacher, M.L. Holmes, A. Newton, P.J. Hogg, L.M. Khachigian, M. Crossley, B.H. Chong, Protein-Protein Interaction between Fli-1 and GATA-1 Mediates Synergistic Expression of Megakaryocyte-Specific Genes through Cooperative DNA Binding, Molecular and Cellular Biology 23 (2003) 3427-3441.

[58] E. Ishiko, I. Matsumura, S. Ezoe, K. Gale, J. Ishiko, Y. Satoh, H. Tanaka, H. Shibayama, M. Mizuki, T. Era, T. Enver, Y. Kanakura, Notch Signals Inhibit the Development of Erythroid/Megakaryocytic Cells by Suppressing GATA-1 Activity through the Induction of HES1, Journal of Biological Chemistry 280 (2005) 4929-4939.

[59] S.J. Loughran, E.A. Kruse, D.F. Hacking, C.A. de Graaf, C.D. Hyland, T.A. Willson, K.J. Henley, S. Ellis, A.K. Voss, D. Metcalf, D.J. Hilton, W.S. Alexander, B.T. Kile, The transcription factor Erg is essential for definitive hematopoiesis and the function of adult hematopoietic stem cells, Nat Immunol 9 (2008) 810-819.

[60] V. Randrianarison-Huetz, B. Laurent, V. Bardet, G.C. Blobe, F. Huetz, D. Duménil, Gfi-1B controls human erythroid and megakaryocytic differentiation by regulating TGF- $\beta$ signaling at the bipotent erythro-megakaryocytic progenitor stage, Blood 115 (2010) 2784-2795.

[61] N.M. Niren, Pharmacologic doses of nicotinamide in the treatment of inflammatory skin conditions: a review, Cutis 77 (2006) 11-16.

[62] J.F. Tallman, S.M. Paul, P. Skolnick, D.W. Gallager, Receptors for the age of anxiety: pharmacology of the benzodiazepines, Science 207 (1980) 274-281.

[63] M. Knip, I.F. Douek, W.P.T. Moore, H.A. Gillmor, A.E.M. McLean, P.J. Bingley, E.A.M. Gale, Safety of high-dose nicotinamide: a review, Diabetologia 43 (2000) 1337-1345. 
[64] Q. Jin, T. Yan, X. Ge, C. Sun, X. Shi, Q. Zhai, Cytoplasm-localized SIRT1 enhances apoptosis, Journal of Cellular Physiology 213 (2007) 88-97.

[65] M.-K. Han, E.-K. Song, Y. Guo, X. Ou, C. Mantel, H.E. Broxmeyer, SIRT1 Regulates Apoptosis and Nanog Expression in Mouse Embryonic Stem Cells by Controlling p53 Subcellular Localization, Cell Stem Cell 2 (2008) 241-251.

[66] T. Peled, H. Shoham, D. Aschengrau, D. Yackoubov, G. Frei, N. Rosenheimer G, B. Lerrer, H.Y. Cohen, A. Nagler, E. Fibach, A. Peled, Nicotinamide, a SIRT1 inhibitor, inhibits differentiation and facilitates expansion of hematopoietic progenitor cells with enhanced bone marrow $\neg$ † homing and engraftment, Experimental Hematology 40 (2012) 342-355.

[67] J.B. Clark, G.M. Ferris, S. Pinder, Inhibition of nuclear NAD nucleosidase and poly ADPribose polymerase activity from rat liver by nicotinamide and 5'-methyl nicotinamide, Biochimica et Biophysica Acta (BBA) - Nucleic Acids and Protein Synthesis 238 (1971) 8285.

[68] Y. Cen, Sirtuins inhibitors: The approach to affinity and selectivity, Biochimica et Biophysica Acta (BBA) - Proteins and Proteomics 1804 (2010) 1635-1644. 


\section{Figure Legends}

Figure 1. Sirtuin expression in K562 cells, according to differentiation state. A) K562 cells differentiate to the $\mathrm{E}$ or MK lineage after treatment with hemin or PMA, respectively. Right panel histogram shows acquisition of CD41 expression after 3 days of PMA treatment, while micrograph shows cells stained positively for hemoglobin after 3 days of hemin treatment. B) Sirtuin expression at the protein level after 3 days of differentiation or not. Representative of two independent experiments. C) Sirtuin expression at the mRNA level after 3 days of differentiation or not $(n=3)$. * indicates $\mathrm{p}<0.05$ compared to SIRT copies in untreated cells by two-tailed t-test with equal variance.

Figure 2. Sirtuin and classical HDAC activity in K562 cells, according to differentiation state. K562 cell lysates were tested for their ability to deacetylate a panel of acetylated peptides using SAMDI. The peptide substrates were of the sequence Ac-GRK ${ }^{\mathrm{Ac}} \mathbf{X Z C}-\mathrm{NH}_{2}$ or Ac-GXK ${ }^{\mathrm{Ac}} \mathbf{Z G C}$ $\mathrm{NH}_{2}$ where $\mathbf{X}$ and $\mathbf{Z}$ represent variable amino acids, and peptides are identified by abbreviated names ( $\mathrm{RK}^{\mathrm{Ac}} \mathbf{X Z}$ or $\mathbf{X K} \mathrm{K}^{\mathrm{Ac}} \mathbf{Z}$ ). The classical HDAC inhibitor TSA was included during lysate-peptide incubations to permit only sirtuin-mediated deacetylation. Nicotinamide was included in lysatepeptide incubations to only permit classical HDAC-mediated deacetylation. The error bars represent the mean $\pm \mathrm{SD}$ of 3 technical replicates, and the presented deacetylase activity is representative of two biological replicates. The deacetylase conversion on each peptide was compared by two-tailed t-test with equal variance. P-values $<0.05$ are indicated for lysates from basal cells compared to hemin-treated (*) and basal cells compared to PMA-treated (\#).

Figure 3. Overexpression of wildtype SIRT1 dramatically reduces $K 562$ cell growth rate. A) Design of the SIRT1 silencing and overexpression lentivectors. B) Lentivector-mediated silencing and overexpression, evaluated by qPCR $(n=2)$. C) The effect of SIRT1 modulation on growth rate over 3 days of culture $(n=3)$. Error bars represent the standard deviation. * indicates $\mathrm{p}<0.05$ compared to empty vector (PWPI) by paired, two-tailed t-test with equal variance. $\alpha$ indicates $\mathrm{p}<0.05$ compared to non-silencing vector (shNS).

Figure 4. SIRT1 promotes erythroid maturation of K562 cells. A) GlyA expression level monitored 4,8 , and 12 days after transduction with wildtype or mutant SIRT1. Representative of 2 
independent time-course experiments. B) Effect of SIRT1 modulation on hemoglobinization as assessed by benzidine staining in untreated or hemin-treated K562 cells $(n=3)$. * indicates $\mathrm{p}<0.05$ compared to empty vector (PWPI) by paired, two-tailed t-test with equal variance. $\alpha$ indicates $\mathrm{p}<0.05$ compared to non-silencing vector (shNS). C) Effect of SIRT1 modulation on globin mRNA expression in untreated K562 cells by qPCR $(n=2)$. Error bars represent the standard deviation.

Figure 5. SIRT1 is essential for efficient PMA-induced MK differentiation of K562 cells. A) Median fluorescence intensity of CD41 during MK differentiation with and without SIRT1 silencing. B) Percentage of polyploid cells during MK differentiation with and without SIRT1 silencing. Error bars represent the standard deviation $(n=3)$. *indicates $p<0.05$ compared to nonsilencing vector (shNS) by two-tailed t-test with equal variance.

Figure 6. SIRT1 is not involved in PMA-induced MK polyploidization. A) CHRF cell MK antigen expression, demonstrating their CD41+CD42- phenotype. B) \% polyploid CHRF cells (control vs shSIRT1-expressing) after induction of polyploidization and maturation by PMA ( $\mathrm{n}=2)$. C) A doxinducible SIRT1 silencing vector was employed to test the effect of changing the timing of silencing relative to the initiation of differentiation in K562 cells. The vector efficiently induced SIRT1 silencing after the addition of dox. D) K562 cells were treated with PMA, and subsequently dox after various time delays, and polyploidization was assessed at Day 7 of PMA $(n=2)$. Error bars represent the standard deviation. * indicates $\mathrm{p}<0.05$ compared to the condition without dox by twotailed t-test with equal variance.

Figure 7. SIRT1 enhances PMA-induced autophagy in K562 cells. A) Comparison of autophagic vacuole formation in K562 cells before and after PMA treatment in control and SIRT1-silenced cells. Representative images of Wright-Giemsa stained ctyospins are shown. Cells were classified as having either no vacuoles, small/punctate vacuoles, or large vacuoles, and the quantification is shown $(\mathrm{n}=3)$. Error bars represent the standard deviation. Number of vacuoles at each day were compared by two-tailed t-test with equal variance. P-values $<0.05$ are indicated for comparisons of the number of large vacuoles (*) and the total number of vacuoles (small + large; \#). B) Cells with either empty vector control or overexpressing SIRT1 were also analyzed for vacuole formation by Wright-Giemsa staining. Representative of two independent experiments. 
Figure 8. SIRT1 exhibits cytosolic localization and enhances LC3 cleavage. A) We analyzed SIRT1 localization in K562 cells prior to treatment or after 2 days of PMA treatment. Substantial levels of SIRT1 expression were detected in the cytosol. Scale bar $=23.6 \mu \mathrm{m}$. B) The effect of SIRT1 modulation on cleavage of LC3. Conversion of LC3-I ( 19 kd) to LC3-II $(\sim 17 \mathrm{kd})$ is a wellaccepted marker of autophagy induction. Cells were treated with PMA and CQ (an inhibitor of autophagasome degradation) for $6 \mathrm{~h}$ prior to harvest of cells for LC3 immunoblotting.

Figure 9. SIRT1 silencing and NIC pretreatment enhance imatinib-induced apoptosis. A) Effects of SIRT1 modulation on the apoptotic response to imatinib treatment. AnnexinV and DAPI stained cells were analyzed to determine the live, apoptotic and dead fractions as shown (n=2). B) NIC dose effects on K562 cell proliferation (n=2). C) NIC dose effects on K562 cell apoptosis after $48 \mathrm{~h}$ $(n=3)$. D) Effect of NIC co-treatment or pretreatment on imatinib-induced apoptosis. Viability and apoptosis were assessed after $48 \mathrm{~h}$ of imatinib treatment by flow cytometry as in (B) $(\mathrm{n}=3)$. Error bars represent the standard deviation. * indicates $\mathrm{p}<0.05$ compared to the condition without NIC by single factor ANOVA using Dunnett's post-hoc test. 

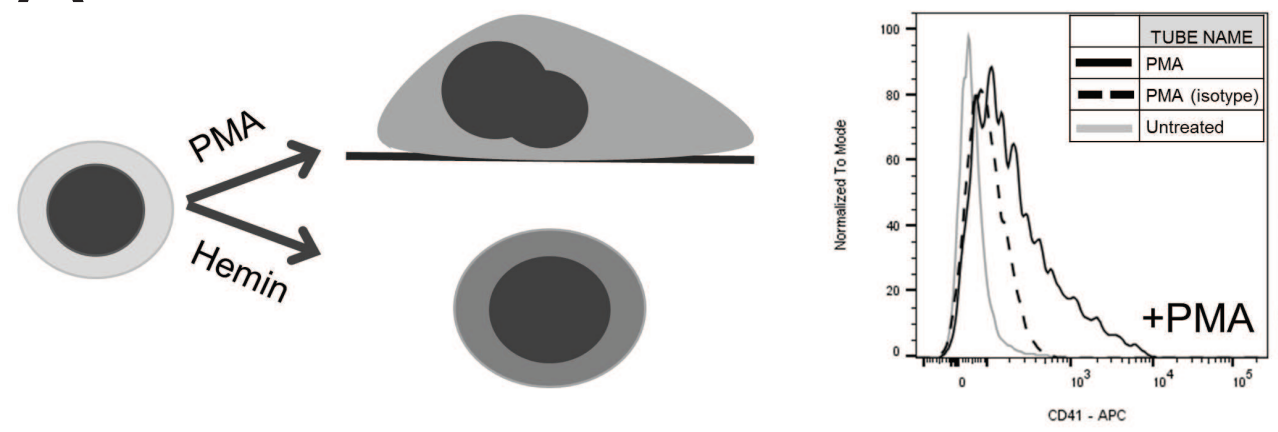

Benzidine staining

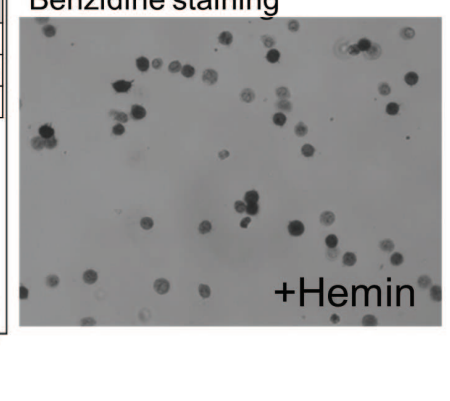

B

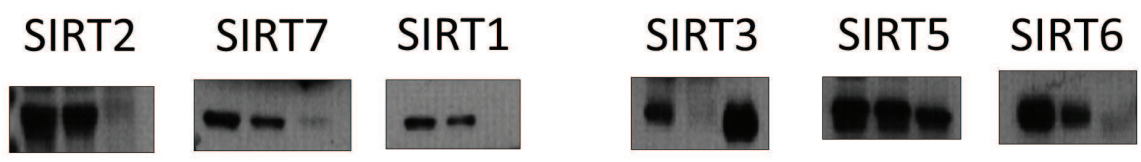

HSC70
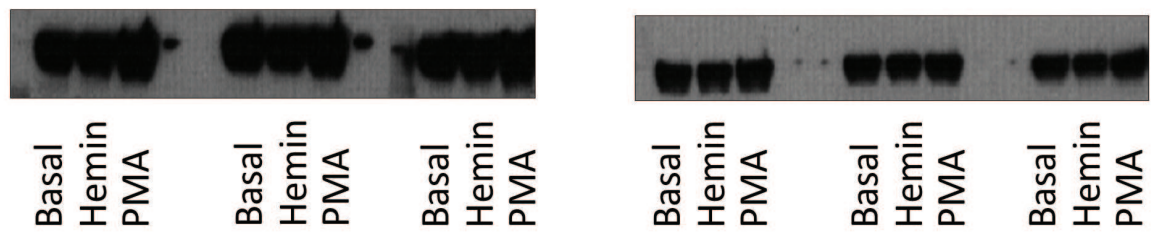

C

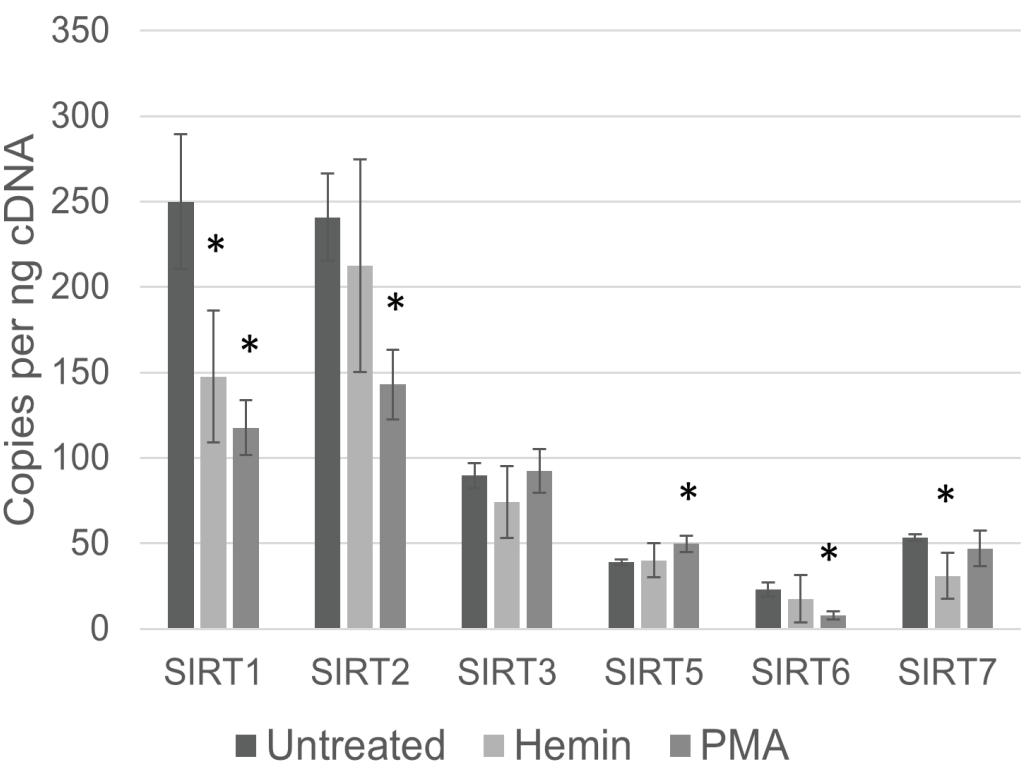



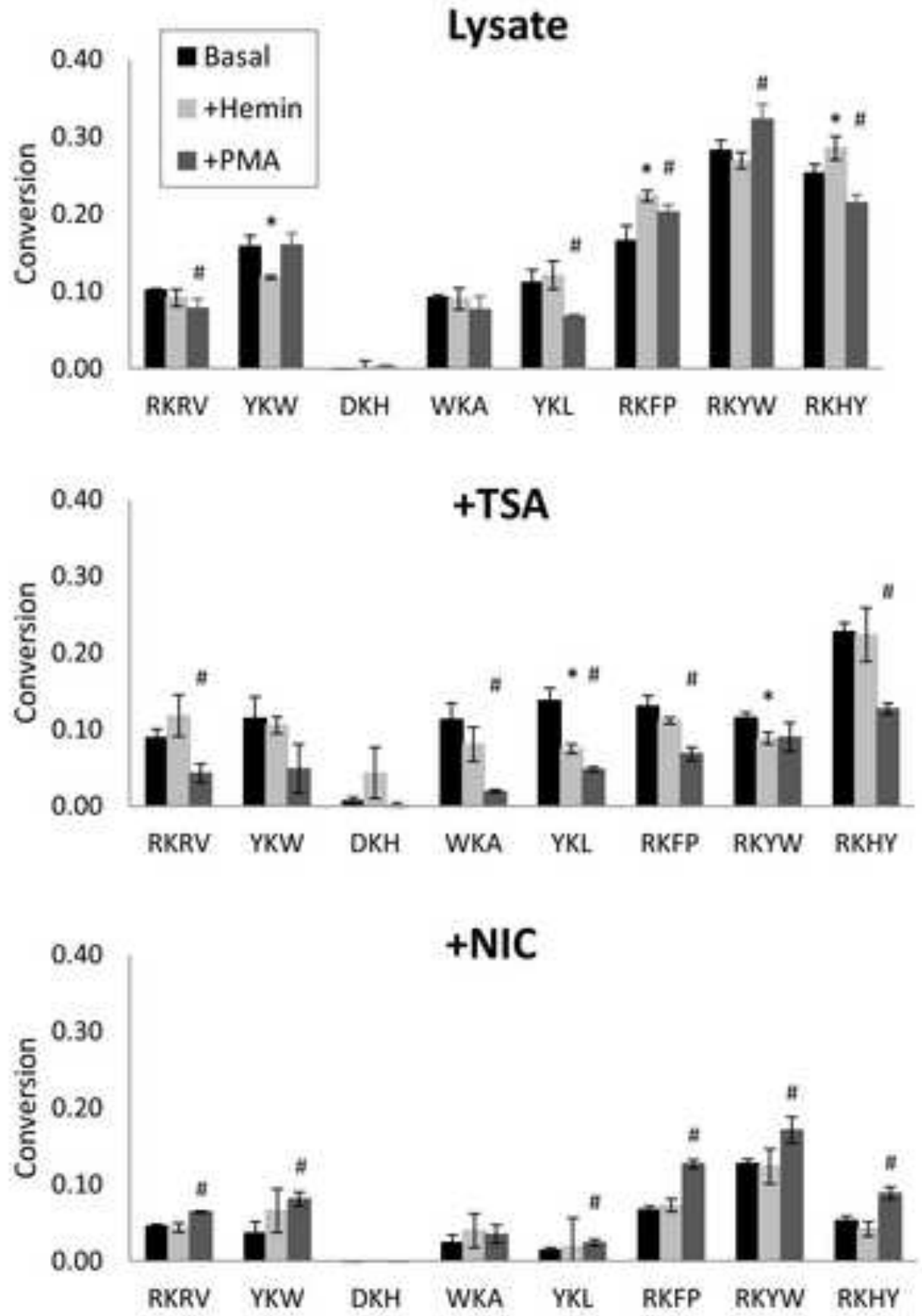
A

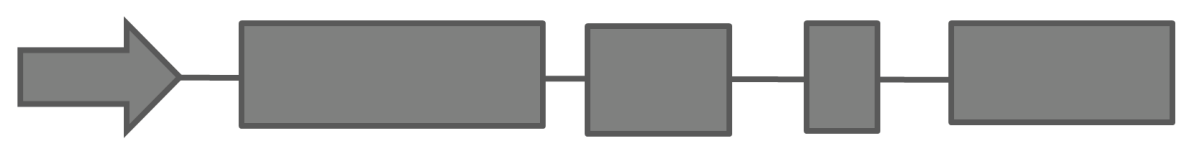

$\begin{array}{llll}\text { EF1- } \alpha & \text { eGFP } & \text { WPRE } & \text { H1 pr. }\end{array}$

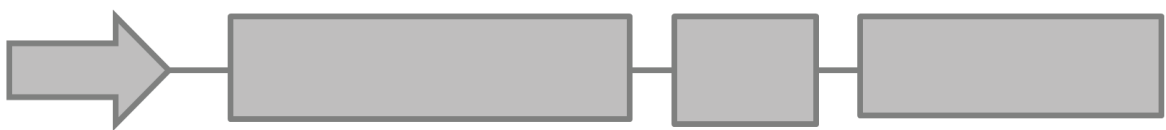

EF1- $\alpha$ SIRT1 (wt or $m t$ IRES eGFP

$\mathrm{B}$
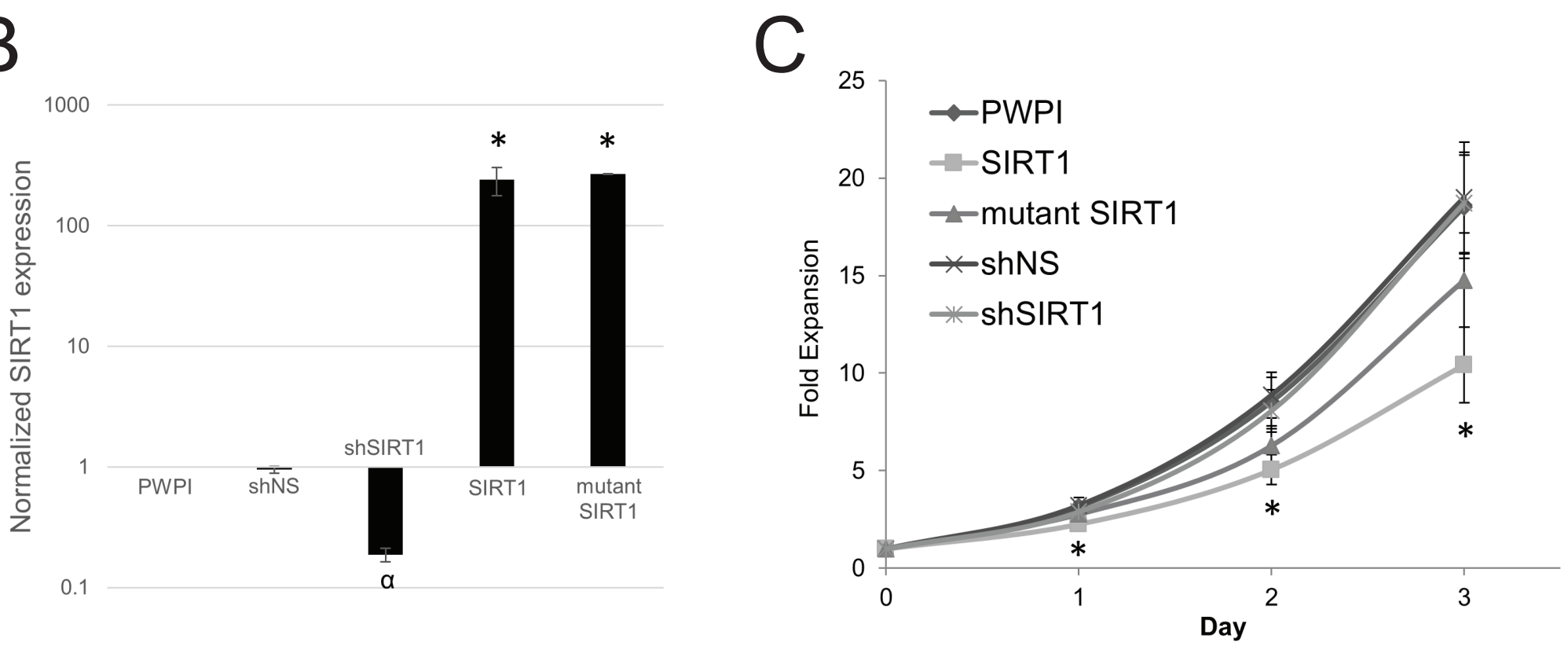
A
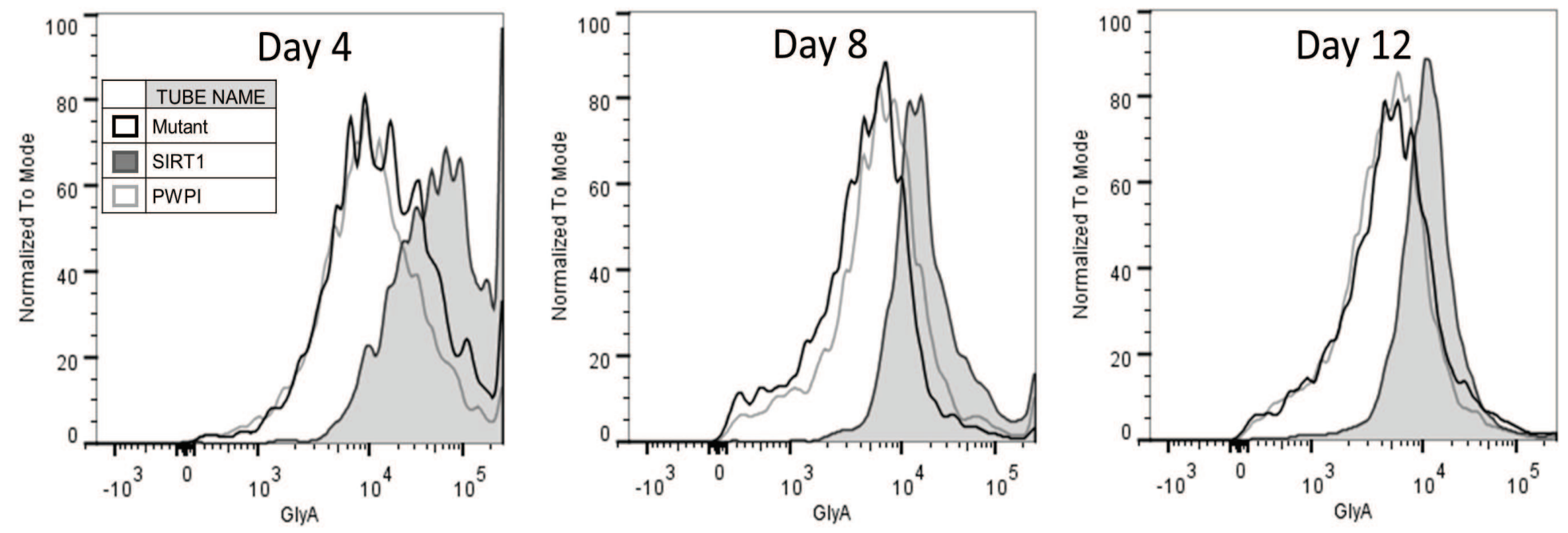

B
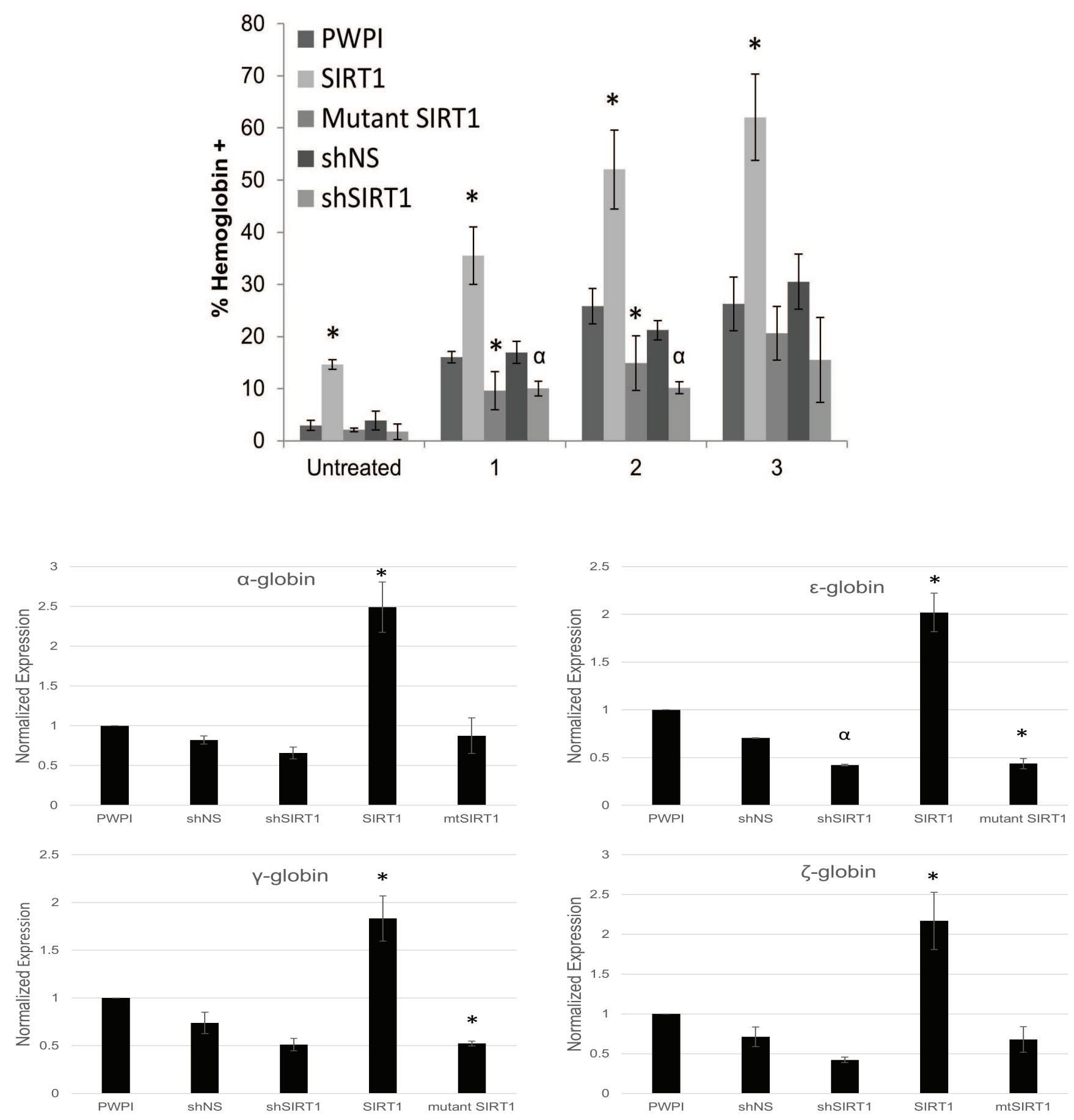
Figure 5

A
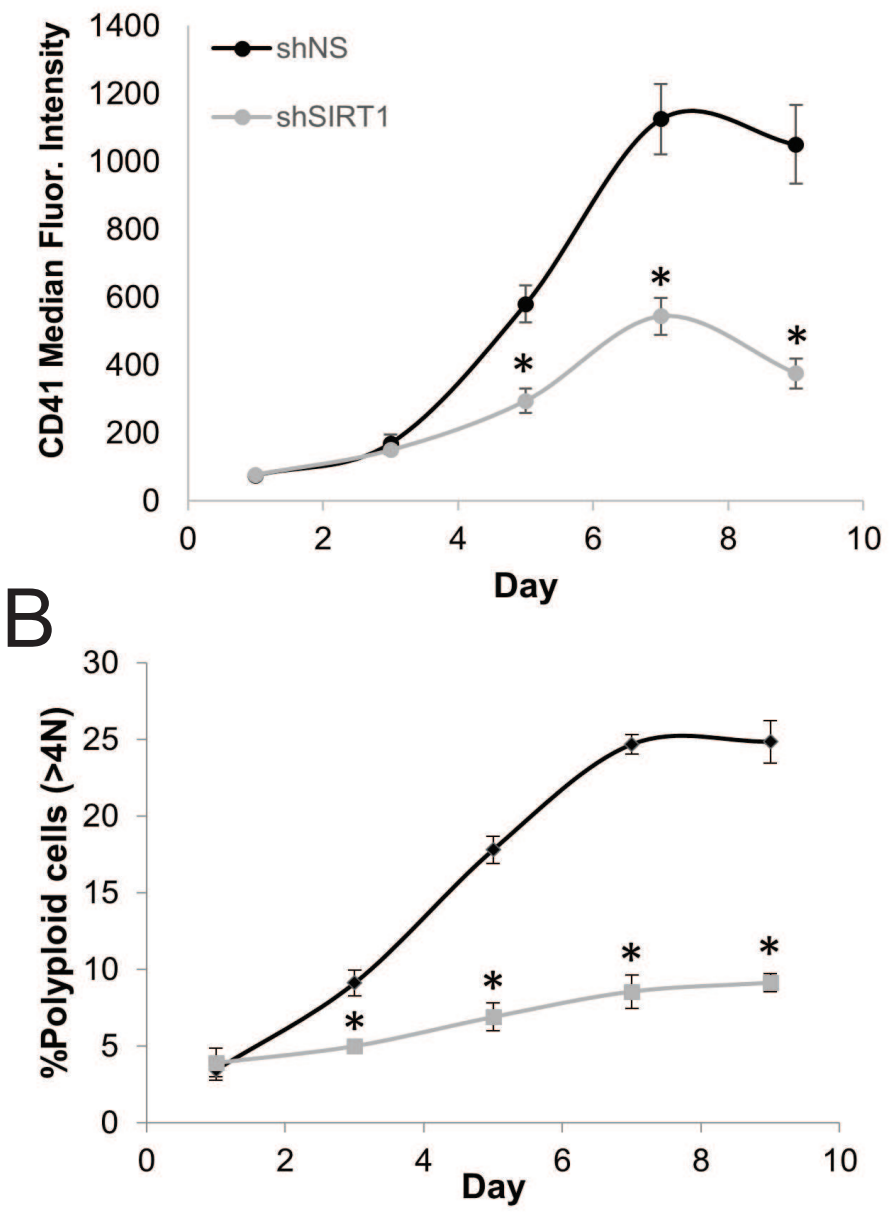

Day 9 CD41 Expression
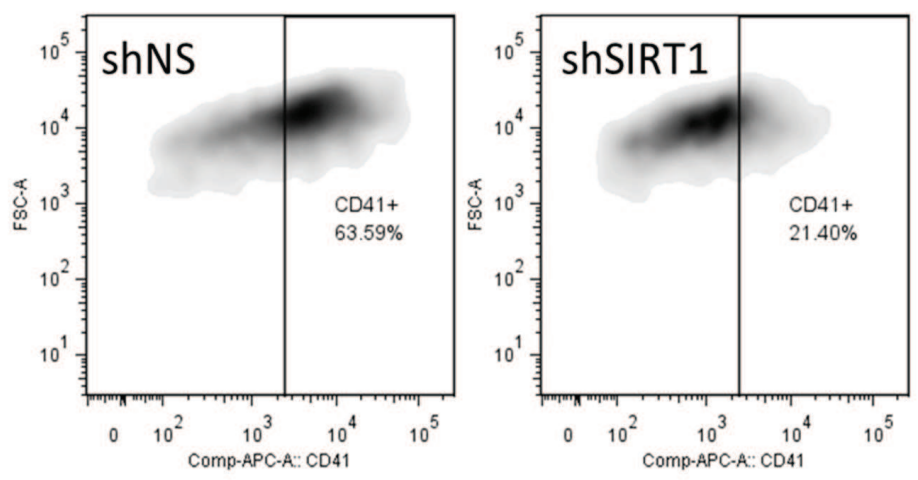

Day 9 Ploidy

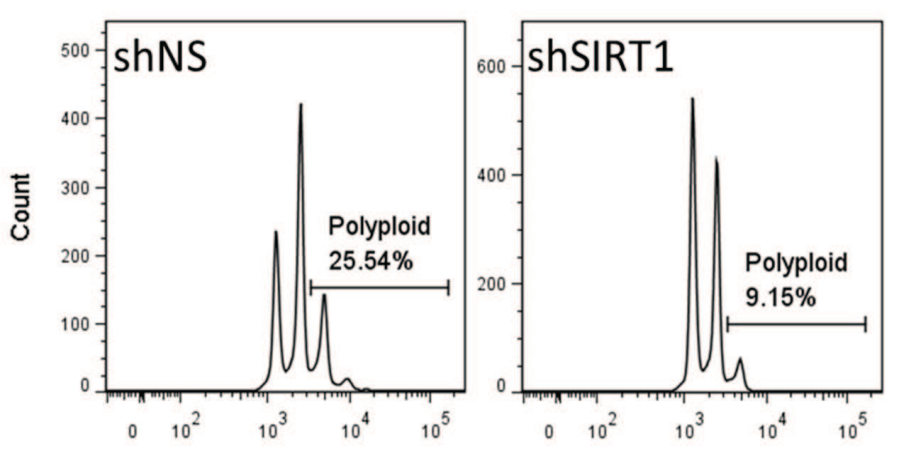


Figure 6
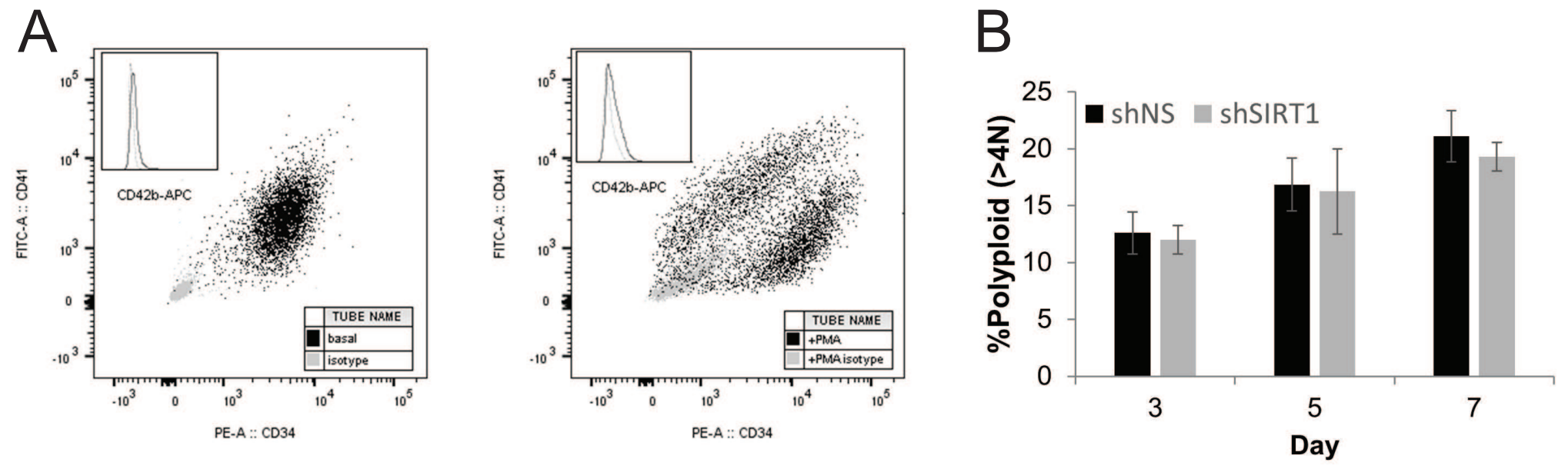

C
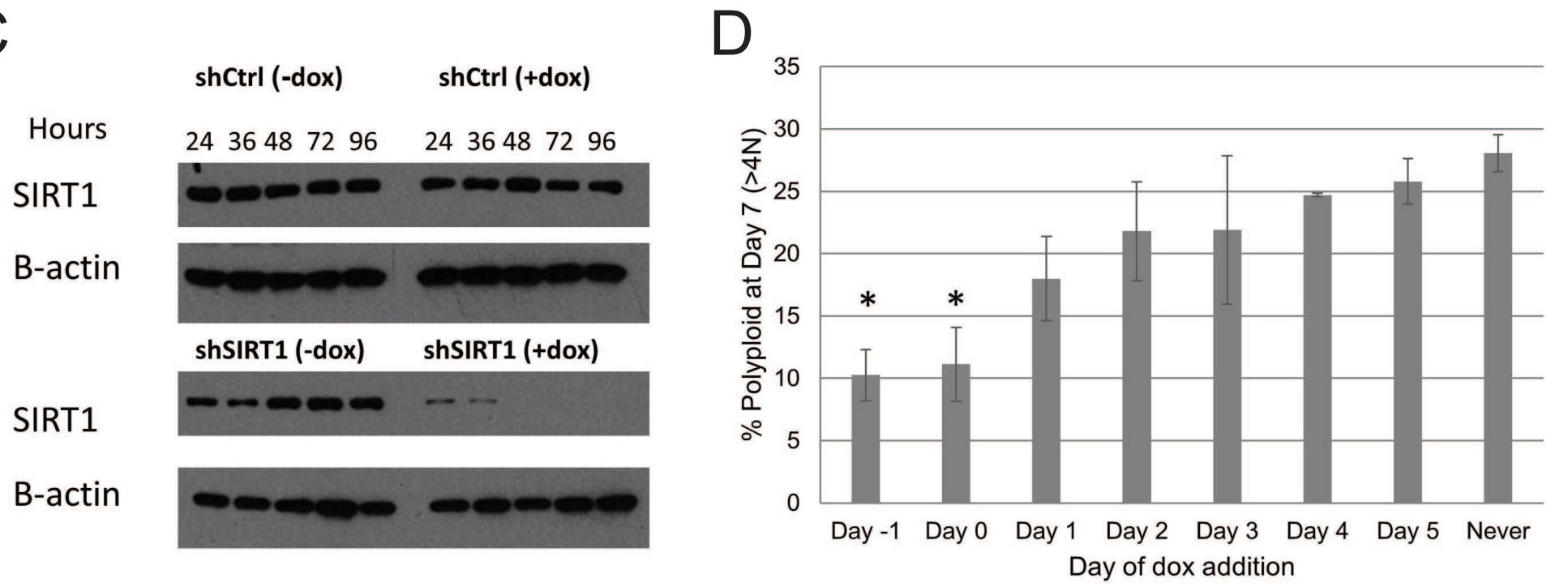

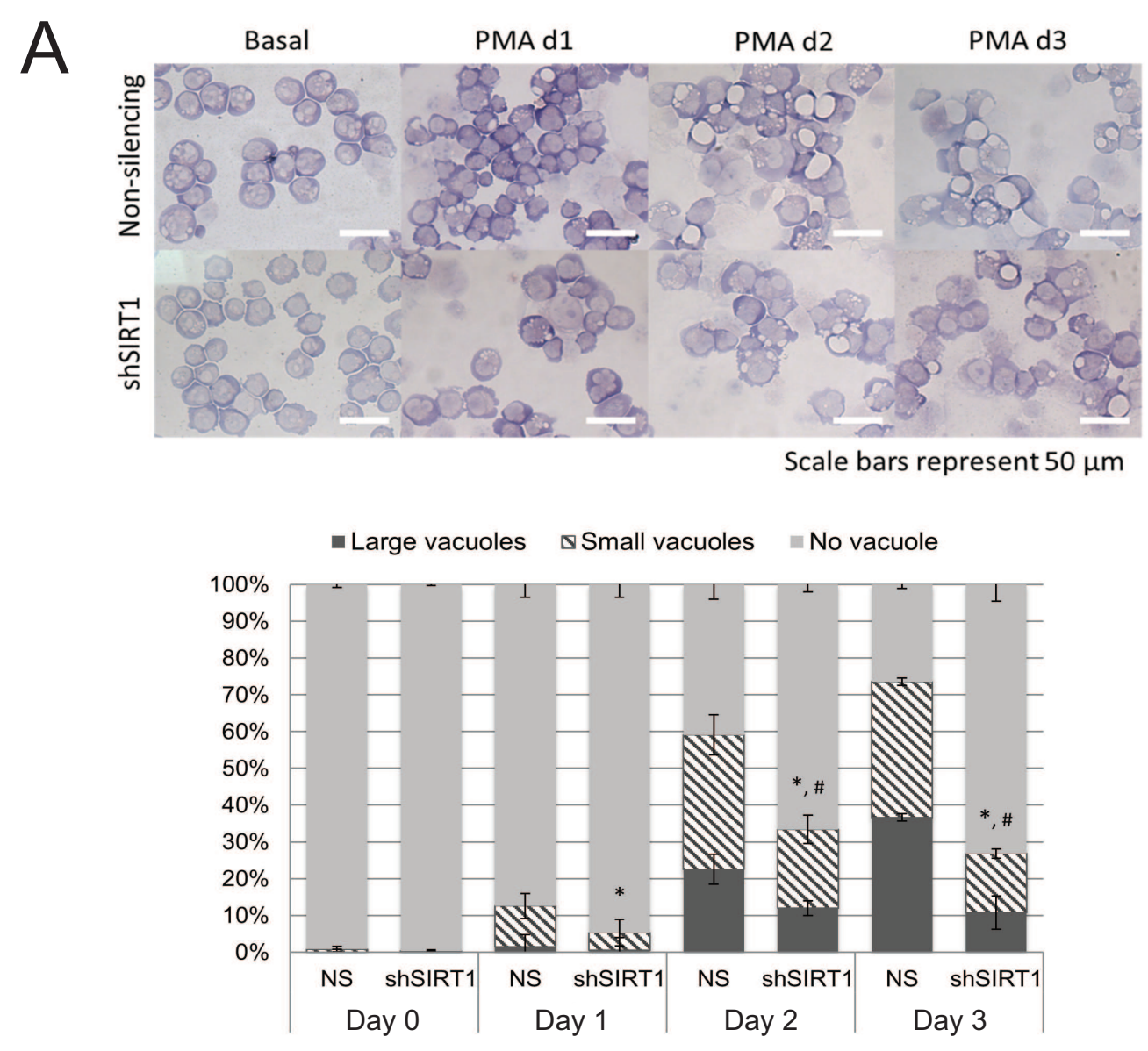

B

Basal

PMA
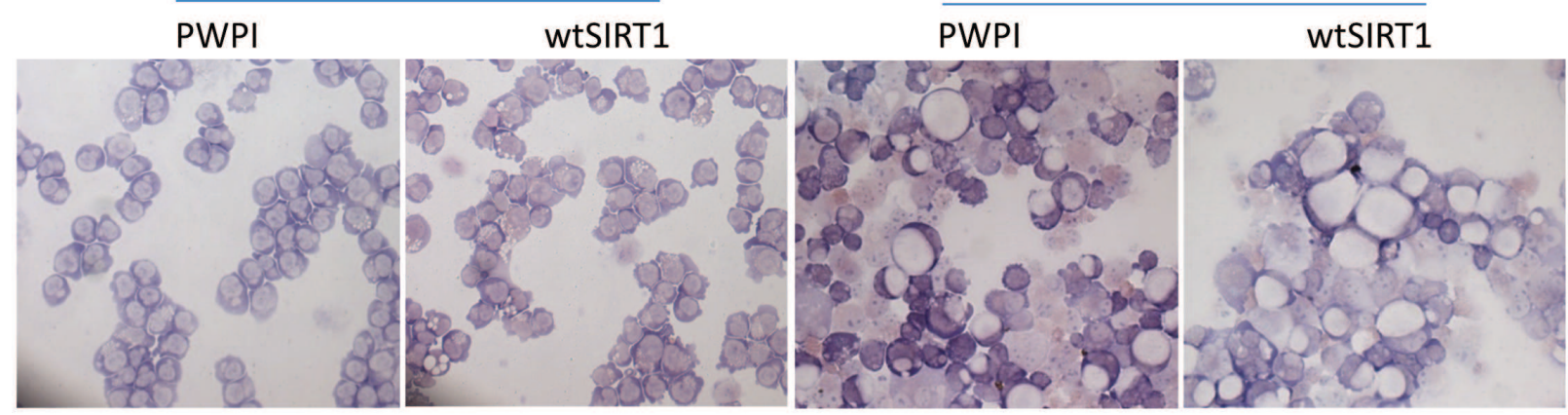
Figure 8

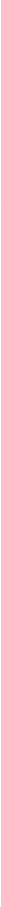




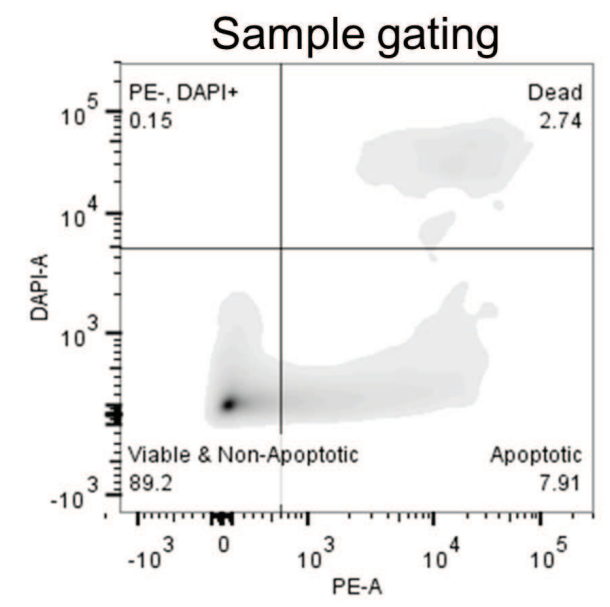

B

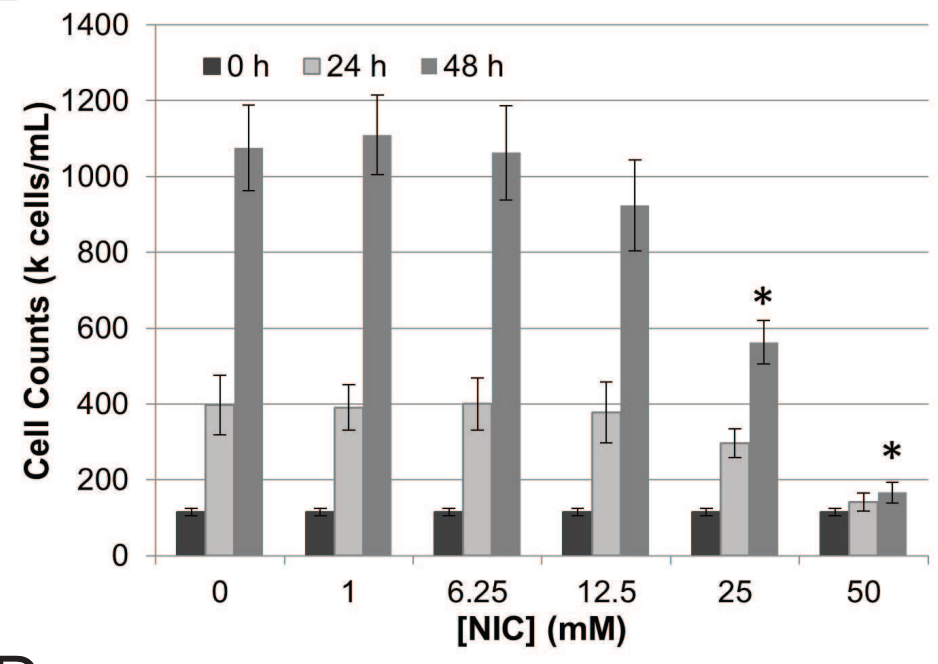

D

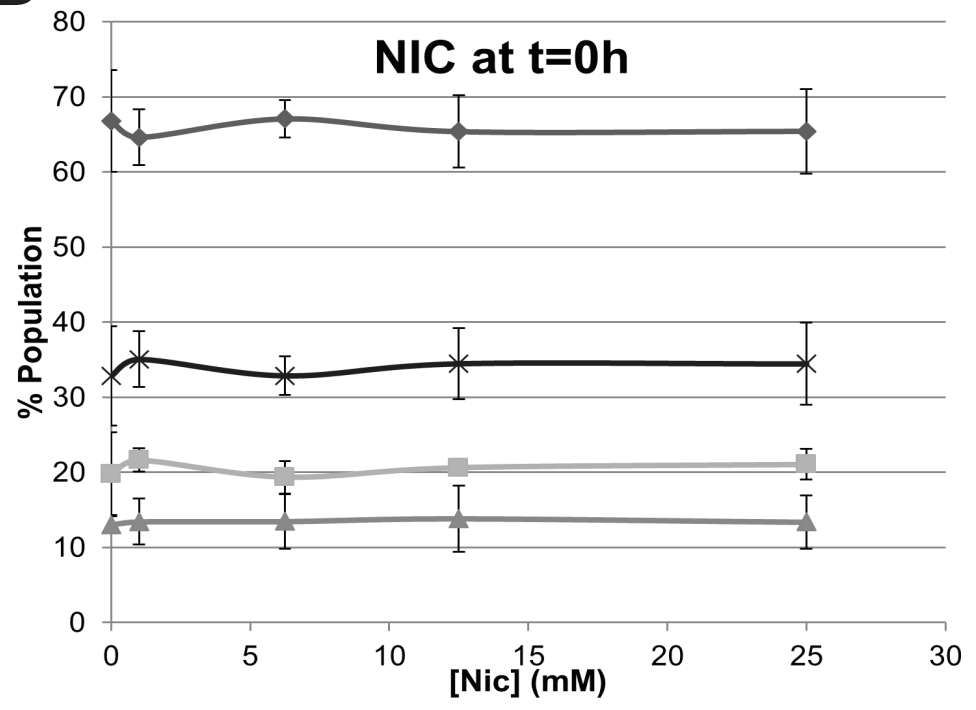

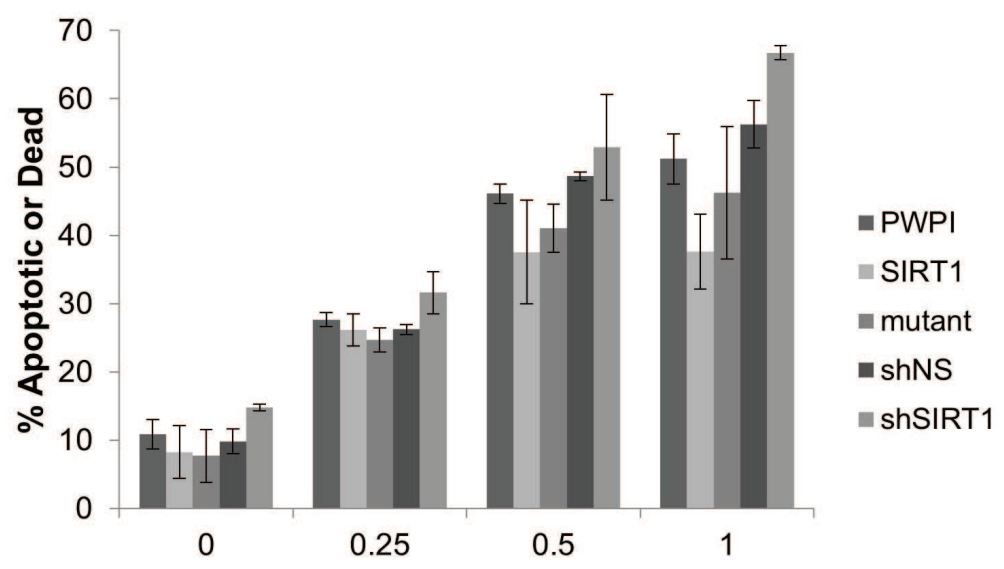

C
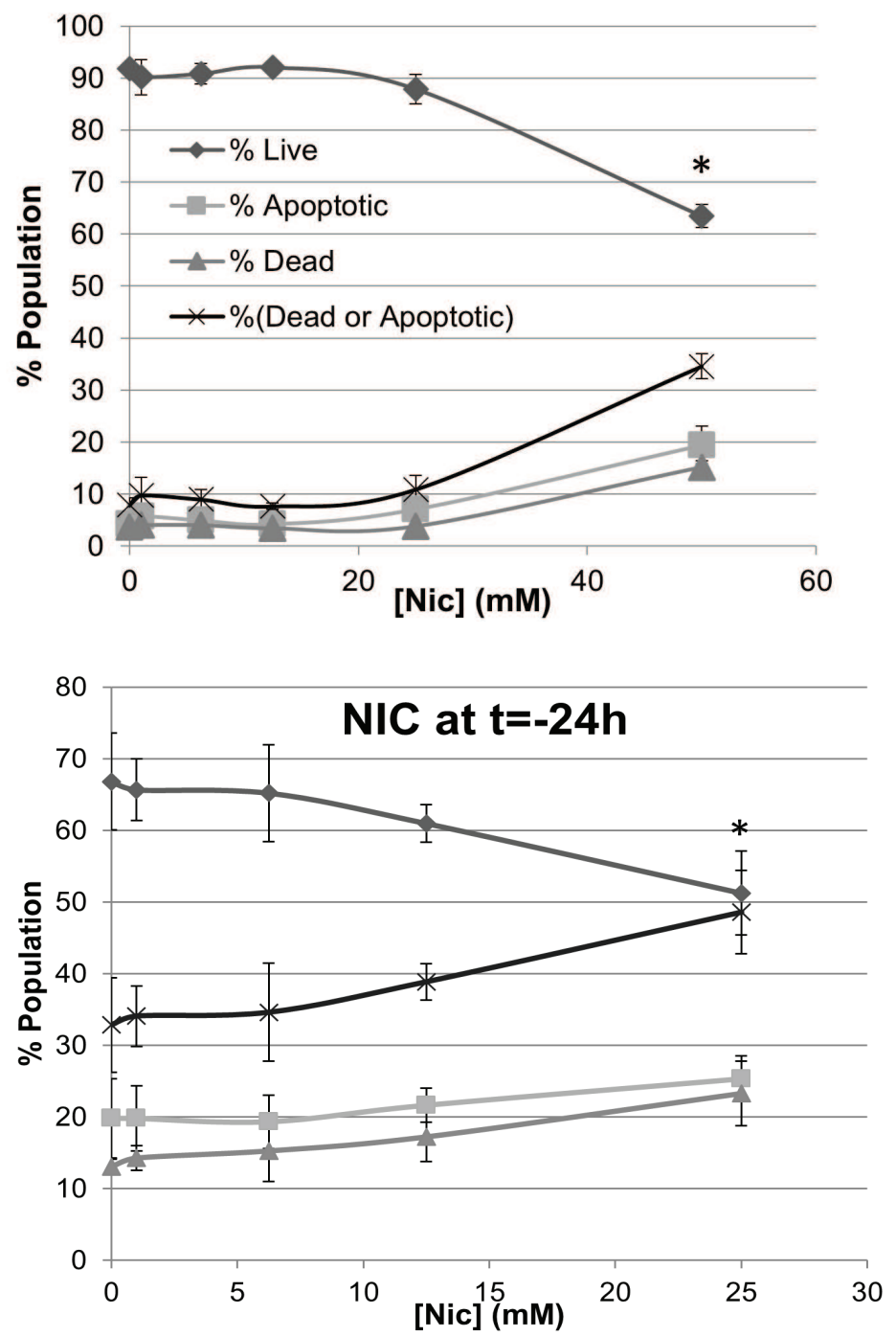